\title{
A STIFFLY STABLE SEMI-DISCRETE SCHEME FOR THE CHARACTERISTIC LINEAR HYPERBOLIC RELAXATION WITH BOUNDARY
}

\author{
Benjamin Boutin*, Thi Hoai Thuong Nguyen and Nicolas Seguin
}

\begin{abstract}
We study the stability of the semi-discrete central scheme for the linear damped wave equation with boundary. We exhibit a sufficient condition on the boundary to guarantee the uniform stability of the initial boundary value problem for the relaxation system independently of the stiffness of the source term and of the space step. The boundary is approximated using a summation-by-parts method and the stiff stability is proved using energy estimates and the Laplace transform. We also investigate if the condition is also necessary, following the continuous case studied by Xin and Xu (J. Differ. Equ. 167 (2000) 388-437).
\end{abstract}

Mathematics Subject Classification. 35F46, 35L50, 65M06, 65M12.

Received May 22, 2019. Accepted February 11, 2020.

\section{INTRODUCTION}

\subsection{Context and motivation}

In many physical situations, we are interested in hyperbolic systems of partial differential equations with relaxation terms [1]. Such systems are found in relaxing gas theory [4], water waves [15,20] and reactive flows [5]. One of the main features of these models is related to the notion of dissipation, leading to smooth solutions and asymptotic stability. The study of the zero relaxation limit for such systems has caught much interest, both from a theoretical and numerical point of view, after the works of Liu [13], Chen et al. [3], Hanouzet and Natalini [8] and Yong [23,24]. In this article, we are concerned with the numerical treatment of the boundary for hyperbolic relaxation systems. Due to the presence of boundary layers and to the possible interaction of the boundary and initial layers, numerical schemes have to be properly designed so as to provide accurate approximations and consistent behaviors.

One of the simplest linear hyperbolic systems with relaxation is the linear damped wave equation on $u^{\varepsilon}, v^{\varepsilon} \in \mathbb{R}$

$$
\left\{\begin{array}{l}
\partial_{t} u^{\varepsilon}+\partial_{x} v^{\varepsilon}=0 \\
\partial_{t} v^{\varepsilon}+a \partial_{x} u^{\varepsilon}=-\varepsilon^{-1} v^{\varepsilon}
\end{array}\right.
$$

Keywords and phrases. Hyperbolic relaxation system, damped wave equation, summation by parts operators, central schemes, energy estimates.

IRMAR (UMR CNRS 6625), Université de Rennes, Campus de Beaulieu, 35042 Rennes Cedex, France.

${ }^{*}$ Corresponding author: benjamin.boutin@univ-rennes1.fr 
where $a>0$ and the relaxation time $\varepsilon>0$ characterizes the stiffness of the relaxation process. When $\varepsilon$ goes to zero, the model may be simplified. We expect indeed that for any position $x$ and any time $t$, the solution $\left(u^{\varepsilon}, v^{\varepsilon}\right)$ tends to $(u(x), 0)$, which is the solution of the corresponding equilibrium system [3,21].

In order to determine a unique solution to the problem (1.1) in the quarter plane $x>0, t>0$, it is necessary to specify values of the solution at initial time

$$
u^{\varepsilon}(x, 0)=u_{0}(x), \quad v^{\varepsilon}(x, 0)=v_{0}(x),
$$

and to impose conditions on the solution at the boundary

$$
B_{u} u^{\varepsilon}(0, t)+B_{v} v^{\varepsilon}(0, t)=b(t),
$$

where $B_{u}$ and $B_{v}$ are constants. For simplicity, we also assume the initial data $f(x)=\left(u_{0}(x), v_{0}(x)\right)$ and the boundary data $b(t)$ to be compatible at the space-time corner $(x, t)=(0,0)$, i.e.

$$
f(0)=f^{\prime}(0)=0, \quad b(0)=b^{\prime}(0)=0 .
$$

In some cases, the suitable boundary conditions comes from physical considerations. At a solid wall that bounds the flow of a fluid, for example, one sets the normal component of the fluid velocity equal to zero (if effects of viscosity are to be considered, the tangential component must also vanish). In other situations, the choice of boundary conditions is not so obvious. This is the case when considering artificial boundaries, which do not correspond to a well-identifies physical phenomenon. In general, not any boundary condition is suitable for a given hyperbolic problem. In the case of the problem (1.1), which is a particular case of the Jin-Xin relaxation model in one space dimension [10], the hyperbolic structure is related to the Riemann invariants $\sqrt{a} u^{\varepsilon} \pm v^{\varepsilon}$ and to the characteristic velocities $\pm \sqrt{a}$. Therefore the boundary condition (1.3) has to satisfy the Uniform Kreiss Condition (UKC)

$$
B_{u}+\sqrt{a} B_{v} \neq 0 .
$$

Only under this assumption, the incoming flow $\sqrt{a} u^{\varepsilon}+v^{\varepsilon}$ at the boundary $x=0$ can be deduced from the outgoing flow $\sqrt{a} u^{\varepsilon}-v^{\varepsilon}$ and the data $b(t)$. Therefore the initial boundary value problem (IBVP) (1.1)-(1.3) is well-posed for each fixed $\varepsilon$ (see $[1,21,22]$ ).

In [21], Xin and $\mathrm{Xu}$ study the asymptotic equivalence of a general linear system of one-dimensional conservation laws and the corresponding relaxation model proposed by Jin and Xin [10] in the limit of a small relaxation rate $\varepsilon$. The main issue is to extend and precise this asymptotic equivalence in the presence of physical boundaries. Within the same problematic, Yong [22] proposed a Generalized Kreiss Condition (GKC) for general multi-dimensional linear constant coefficient relaxation systems, or one-dimensional nonlinear systems, with non-characteristic boundaries. This condition enables uniform stability estimates and a reduced boundary condition for the corresponding equilibrium system. For the special Jin-Xin system (1.1) with boundary condition (1.3) but with stiff source terms of the form $\varepsilon^{-1}\left(\lambda u^{\varepsilon}-v^{\varepsilon}\right)$ for some $\lambda$, Xin and Xu identify and rigorously justify a necessary and sufficient condition (which they call the Stiff Kreiss Condition, or SKC in short) on the boundary condition to guarantee the uniform well-posedness of the IBVP, independently of the relaxation parameter. In addition to the work in [22], their study also covers the characteristic case and provides optimal asymptotic expansions for the limit process, handling with boundary and/or initial layers. In the case of our system (1.1), the parameter $\lambda=0$ so that the boundary is characteristic for limit equation, and the SKC in [21] then simply reduces to

$$
B_{v}=0, \quad \text { or } \quad \frac{B_{u}}{B_{v}} \notin[-\sqrt{a}, 0] .
$$

The motivation of the present study is to analyze the counterpart of the above results but now for the difference approximation of the IBVP (1.1)-(1.3). The major issues in the theory of the relaxation approximations to equilibrium system of conservation laws is the appearance of stiff boundary layers in the presence of physical or numerical boundary conditions due to the additional characteristic speeds introduced in the relaxation systems. 
On the other hand, the stability estimate obtained for a certain approximation is the key to the proper error estimates. Thus, the way of formulating boundary conditions for the relaxation systems so as to guarantee the uniform stability and to minimize the artificial boundary layer is a crucial issue to the success of the schemes.

\subsection{Description of the semi-discrete numerical scheme}

Let $\Delta x>0$ be the space step and $U\left(x_{j}, t\right)=\left(u^{\varepsilon}, v^{\varepsilon}\right)^{T}\left(x_{j}, t\right)$ with $x_{j}=j \Delta x$, for any $j \in \mathbb{N}$. The solution to the $\operatorname{IBVP}(1.1)-(1.3)$ is approximated by a sequence $U_{j}(t)=\left(u_{j}(t), v_{j}(t)\right)^{T}$ (where we omit the explicit dependence on $\varepsilon$ ). We focus in this paper on the semi-discrete approximation of the IBVP obtained by the central differencing scheme and derive a sufficient condition for its stiff stability. Let $A, S$ and $B$ be the following matrices:

$$
A=\left(\begin{array}{cc}
0 & 1 \\
a & 0
\end{array}\right), \quad S=\left(\begin{array}{cc}
0 & 0 \\
0 & -1
\end{array}\right), \quad B=\left(B_{u} B_{v}\right) .
$$

A first step towards the semi-discrete approximation of the IBVP (1.1)-(1.3) is the following system

$$
\begin{aligned}
\partial_{t} U_{j}(t)+(\mathcal{Q} U)_{j}(t) & =\frac{1}{\varepsilon} S U_{j}(t), \quad j \geq 1, \\
U_{j}(0) & =f_{j}, \\
B U_{0}(t) & =b(t),
\end{aligned}
$$

with the discrete Cauchy data $f_{j}=U\left(x_{j}, 0\right)$. The difference operator $(\mathcal{Q} U)_{j}(t)$ is a consistent approximation of the first order space-derivative $A \partial_{x} U\left(x_{j}, t\right)$ in the sense that $(\mathcal{Q} U)\left(x_{j}, t\right)=A \partial_{x} U\left(x_{j}, t\right)+\mathcal{O}\left(\Delta x^{p}\right)$, for some $p>0$. It is defined at any discrete point including the boundary point $j=0$.

The summation by parts (SBP) finite difference operators were first derived in $[11,12]$. In [16], the analysis was revisited and exact expressions for the finite difference coefficients were obtained. In the case of the central scheme, the modification of the difference operator $\mathcal{Q} U$ at $j=0$ can also be interpreted as the use of an extra boundary condition. It means that we use the centered approximation at the boundary point $j=0$ but supply another boundary condition that determines a ghost value $U_{-1}$ through the identity

$$
U_{1}-2 U_{0}+U_{-1}=0
$$

If we eliminate $U_{-1}$, then we obtain a one-sided approximation. In [7], the corresponding energy estimate is obtained by using the scalar product and norm

$$
\langle U, V\rangle_{\Delta x}=\frac{\Delta x}{2}\left\langle U_{0}, V_{0}\right\rangle+\Delta x \sum_{j=1}^{\infty}\left\langle U_{j}, V_{j}\right\rangle, \quad\|U\|_{\Delta x}^{2}=\langle U, U\rangle_{\Delta x} .
$$

with $\langle.,$.$\rangle being the usual Euclidean inner product. The considered difference operator reads$

$$
(\mathcal{Q} U)_{j}= \begin{cases}\frac{1}{2 \Delta x} A\left(U_{j+1}-U_{j-1}\right), & j \geq 1, \\ \frac{1}{\Delta x} A\left(U_{1}-U_{0}\right), & j=0,\end{cases}
$$

which uses a noncentered approximation at the boundary, so that the difference operator is defined at all gridpoints including the boundary point $j=0$.

Let us emphasize that the numerical scheme (1.7) still needs one more scalar equation at the boundary point $j=0$ so as to be fully defined, due to the fact that the matrix $B$ has rank one. This is actually a discrete feature only, since in the continuous case this single equation is exactly complemented by the only incoming characteristic (under UKC). We choose to define the remaining discrete boundary value in agreement with the 
dissipativity of the source term. We then use a symmetric form of the problem, based on the matrix $P$ and on the symmetric positive definite matrix $H_{P}$ below

$$
P=\left(\begin{array}{cc}
B_{u} & B_{v} \\
1 & 0
\end{array}\right), \quad H_{P}=\left(\begin{array}{cc}
1 & -B_{u} \\
-B_{u} & a B_{v}^{2}+B_{u}^{2}
\end{array}\right) .
$$

As a consequence, the matrix $P^{T} H_{P} P$ is symmetric positive definite, $H_{P} P A P^{-1}$ is symmetric and $H_{P} P S P^{-1}$ is negative definite. Since $P^{T} H_{P} P A$ is also a symmetric matrix, one has

$$
\left\langle U, P^{T} H_{P} P(\mathcal{Q} U)\right\rangle_{\Delta x}(t)=-\frac{1}{2}\left\langle U_{0}, P^{T} H_{P} P A U_{0}\right\rangle(t),
$$

which is the discrete counterpart of the equality $\int_{0}^{+\infty}\left\langle U, P^{T} H_{P} P A \partial_{x} U\right\rangle(x, t) \mathrm{d} x=-\frac{1}{2}\left\langle U, P^{T} H_{P} P A U\right\rangle(0, t)$ available in the continuous case. Moreover, at the boundary $j=0$, we obtain

$$
\left\langle\partial_{t} U_{0}, P^{T} H_{P} P U_{0}\right\rangle(t)+\left\langle(\mathcal{Q} U)_{0}, P^{T} H_{P} P U_{0}\right\rangle(t)=\frac{1}{\varepsilon}\left\langle S U_{0}, P^{T} H_{P} P U_{0}\right\rangle(t) .
$$

Inserting now the homogeneous boundary condition $B U_{0}(t)=0$ and introducing the matrix $\Pi_{2}=\left(\begin{array}{ll}0 & 1\end{array}\right)$, the previous equality (1.10) can be reformulated as

$$
\partial_{t}\left(\Pi_{2} H_{P} P U_{0}\right)(t)\left(\Pi_{2} P U_{0}\right)(t)+\left(\Pi_{2} H_{P} P(\mathcal{Q U})_{0}\right)(t)\left(\Pi_{2} P U_{0}\right)(t)=\frac{1}{\varepsilon}\left(\Pi_{2} H_{P} P S U_{0}\right)(t)\left(\Pi_{2} P U_{0}\right)(t) .
$$

We therefore propose the following numerical approximation at the boundary

$$
\partial_{t}\left(\Pi_{2} H_{P} P U_{0}\right)(t)+\Pi_{2} H_{P} P(\mathcal{Q U})_{0}(t)=\frac{1}{\varepsilon} \Pi_{2} H_{P} P S U_{0}(t) .
$$

To summarize, along the rest of the paper, we will study the following semi-discrete approximation of the IBVP (1.1)-(1.3):

$$
\begin{cases}\partial_{t} U_{j}(t)+(\mathcal{Q} U)_{j}(t)=\varepsilon^{-1} S U_{j}(t), & j \geq 1, t \geq 0 \\ U_{j}(0)=f_{j}, & j \geq 0, \\ B U_{0}(t)=b(t), & t \geq 0, \\ \partial_{t}\left(\Pi_{2} H_{P} P U_{0}\right)(t)+\Pi_{2} H_{P} P(\mathcal{Q} U)_{0}(t)=\varepsilon^{-1} \Pi_{2} H_{P} P S U_{0}(t), & t \geq 0\end{cases}
$$

\subsection{Main result}

For the continuous IBVP (1.1)-(1.3), the UKC (1.5) is not enough and a more stringent restriction has to be imposed. Our aim is to determine a sufficient condition for the stiff stability of the above semi-discrete IBVP (1.11), in other words the uniform stability with respect to the stiffness of the relaxation term. First of all, let us address the question of the existence and uniqueness of a solution to the infinite-dimensional ODE system (1.11) through the next result.

Lemma 1.1. Let us consider some fixed parameters $\left(B_{u}, B_{v}\right) \in \mathbb{R}^{2}$ with $B_{v} \neq 0$, and $\varepsilon, \Delta x>0$. For any $\left(f_{j}\right)_{j \in \mathbb{N}} \in l^{2}\left(\mathbb{N}, \mathbb{R}^{2}\right)$ and any $b \in \mathcal{C}^{1}\left(\mathbb{R}_{+}, \mathbb{R}\right)$ there exists a unique solution $\left(U_{j}\right)_{j \in \mathbb{N}} \in \mathcal{C}^{1}\left(\left[0,+\infty\left[, l^{2}\left(\mathbb{N}, \mathbb{R}^{2}\right)\right)\right.\right.$ to $(1.11)$.

Proof. The proof rests on the common linear Cauchy-Lipschitz theorem in the Banach space $l^{2}\left(\mathbb{N}, \mathbb{R}^{2}\right)$. Let us bring some precisions concerning the solvability of the two rank-one boundary equations. The first algebraic equation reads simply

$$
B_{u} u_{0}(t)+B_{v} v_{0}(t)=b(t),
$$


while the second differential one reads equivalently, for some linear operator $L: \mathbb{R}^{2} \times \mathbb{R}^{2} \rightarrow \mathbb{R}$, as

$$
a B_{v}^{2} u_{0}^{\prime}(t)-B_{u} B_{v} v_{0}^{\prime}(t)+\mathrm{L}\left(U_{0}(t), U_{1}(t)\right)=0 .
$$

Eliminating $v_{0}$ from the algebraic boundary condition, we get thus $B_{v} v_{0}^{\prime}(t)=b^{\prime}(t)-B_{u} u_{0}^{\prime}(t)$ and therefore

$$
\left(a B_{v}^{2}+B_{u}^{2}\right) u_{0}^{\prime}(t)=-\mathrm{L}\left(U_{0}(t), U_{1}(t)\right)+B_{u} b^{\prime}(t) .
$$

The solvability of the whole ODE system is therefore deduced by $B_{v} \neq 0$ together with $a B_{v}^{2}+B_{u}^{2} \neq 0$. The details are left to the reader.

Theorem 1.2 (Main result). Under the strict dissipativity condition

$$
B_{u} B_{v}>0,
$$

for any $T>0$ there exists $C_{T}>0$ such that for any $\left(f_{j}\right)_{j \in \mathbb{N}} \in l^{2}\left(\mathbb{N}, \mathbb{R}^{2}\right)$ and any $b \in \mathcal{C}^{1}\left(\mathbb{R}^{+}, \mathbb{R}\right) \cap L^{2}\left(\mathbb{R}^{+}, \mathbb{R}\right)$, the solution $\left(U_{j}\right)_{j \in \mathbb{N}}$ to (1.11) satisfies

$$
\int_{0}^{T}\left|U_{0}(t)\right|^{2} \mathrm{~d} t+\int_{0}^{T} \sum_{j \geq 0} \Delta x\left|U_{j}(t)\right|^{2} \mathrm{~d} t \leq C_{T}\left(\sum_{j \geq 0} \Delta x\left|f_{j}\right|^{2}+\int_{0}^{T}|b(t)|^{2} \mathrm{~d} t\right),
$$

where the constant $C_{T}$ is independent of the data $f$ and $b$, but most importantly of $\varepsilon \in(0,+\infty)$ and $\Delta x \in(0,1]$.

The proof of Theorem 1.2 is based on two main ingredients, by assembling a result for the case of homogeneous boundary data and another for the case with homogeneous initial data. We state successively hereafter these two statements.

Proposition 1.3 (Homogeneous boundary condition). Assume that the parameters $\Delta x \in(0,1], \varepsilon>0$ and $\left(B_{u}, B_{v}\right)$ satisfy the discrete strict dissipativity condition

$$
2 a \frac{B_{u}}{B_{v}}+\frac{\Delta x}{\varepsilon}\left(\frac{B_{u}}{B_{v}}\right)^{2}>0
$$

Then there exists a constant $c>0$ such that for any $\left(f_{j}\right)_{j \in \mathbb{N}} \in l^{2}\left(\mathbb{N}, \mathbb{R}^{2}\right)$, the solution $\left(U_{j}\right)_{j \in \mathbb{N}}$ to (1.11) with $b \equiv 0$ satisfies

$$
\left\langle U, P^{T} H_{P} P U\right\rangle_{\Delta x}(T)+c \int_{0}^{T}\left|U_{0}\right|^{2}(t) \mathrm{d} t \leq\left\langle f, P^{T} H_{P} P f\right\rangle_{\Delta x}
$$

More precisely,

(a) If $B_{u} B_{v}>0$, then (1.15) holds uniformly, i.e. with $c$ independent of $\varepsilon$ and $\Delta x$.

(b) If $B_{u} B_{v}<0$, then considering some $\delta_{0}>-2 a B_{v} B_{u}^{-1}$, there exists $c\left(\delta_{0}\right)>0$ such that $(1.15)$ holds uniformly with $c=c\left(\delta_{0}\right)$, as soon as $\Delta x \geq \delta_{0} \varepsilon$.

Proposition 1.4 (Homogeneous initial condition). Assume that the boundary condition is strictly dissipative, thus satisfying (1.12). Then, there exists a constant $c>0$ such that for any $\alpha>0$ there exists $\Delta x_{0}>0$ such that the following property holds. For any $b \in \mathcal{C}^{1}\left(\mathbb{R}^{+}, \mathbb{R}\right) \cap L^{2}\left(\mathbb{R}^{+}, \mathbb{R}\right)$ and $\Delta x \leq \Delta x_{0}$, the solution $\left(U_{j}\right)_{j \in \mathbb{N}}$ to (1.11) with $\left(f_{j}\right)_{j \in \mathbb{N}} \equiv 0$ satisfies

$$
\alpha \Delta x \int_{0}^{\infty} \sum_{j \geq 0} \mathrm{e}^{-2 \alpha t}\left|U_{j}(t)\right|^{2} \mathrm{~d} t+\int_{0}^{\infty} \mathrm{e}^{-2 \alpha t}\left|U_{0}(t)\right|^{2} \mathrm{~d} t \leq c \int_{0}^{\infty} \mathrm{e}^{-2 \alpha t}|b(t)|^{2} \mathrm{~d} t .
$$


Remark 1.5. Since $P^{T} H_{P} P$ is a symmetric positive definite matrix, the following inequality holds for some constants $m, n>0$, independent of $\Delta x$ :

$$
m\left\langle U, P^{T} H_{P} P U\right\rangle_{\Delta x}(t) \leq\langle U, U\rangle_{\Delta x}(t) \leq n\left\langle U, P^{T} H_{P} P U\right\rangle_{\Delta x}(t),
$$

which will be useful to prove the estimate (1.13) with weighted-in-time norms from (1.15).

Xin and Xu [21] considered the IBVP for the Jin-Xin relaxation model [10] and derived the SKC (1.6) for its stiff well-posedness. They show in particular that the IBVP is well-posed if and only if (1.6) holds. In the discrete IBVP (1.11), it seems that even the SKC is not sufficient to derive uniform stability estimates. In comparison, the strict dissipativity condition (1.12) is more restrictive, but we are only able to prove that it is sufficient. Following [21,22], we also perform a normal mode analysis to construct unstable solutions and, based on some numerical investigations, condition (1.12) would appear to be also necessary for the stiff stability. Let us mention that the discrete strict dissipativity condition (1.14) is not implied by the SKC (1.6), probably due to some numerical diffusion at the boundary.

The Proposition 1.3 is studied in Section 2.1 by means of the discrete energy method. In order to illustrate the relevance of the condition (1.14), we present in Section 2.2 some numerical results, for various values of the parameters $\left(B_{u}, B_{v}\right)$ and show that the energy $\left\langle U(t), P^{T} H_{P} P U(t)\right\rangle_{\Delta x}$ increases if the condition (1.14) does not hold. In Section 3.1, we want to address the question of the existence of unstable solutions in order to derive necessary condition for stability by using the normal mode analysis. In Section 3.2, we present numerical results and show that $B_{u} B_{v}>0$ seems to be necessary to ensure the stiff stability of the discrete IBVP. Even if the boundary condition (1.3) satisfies the SKC, there exist unstable solutions of the discrete IBVP (1.11). To isolate the effects of a possible boundary layer and avoid the complicated interactions of boundary and initial layers, in Section 4, we consider the IBVP (1.11) with homogeneous initial data and nonzero boundary data $b(t)$. In Section 4.1, the numerical solution $\left(U_{j}(t)\right)_{j \in \mathbb{N}}$ can be constructed by Laplace transform. By using the Parseval's identity, under assumption $B_{u} B_{v}>0$, the Proposition 1.4 is proved in Section 4.2. In Appendix A, we study an example of how waves occur in modeling the action of an elastic string over time, which is a particular case of linear damped wave equation. By using the Newton's Second Law of Motion, we can derive the boundary condition $B_{u} B_{v}>0$ for this system.

\section{Stiff Stability of the SEMi-Discrete IBVP With homogeneous Boundary CONDITION}

In this section, we consider the IBVP (1.11) for homogeneous boundary condition $b \equiv 0$, nonzero Cauchy data $\left(f_{j}\right)_{j \in \mathbb{N}} \in l^{2}\left(\mathbb{N}, \mathbb{R}^{2}\right)$ and prove Proposition 1.3 by means of the discrete energy method.

\subsection{The energy method}

In the continuous case, the energy estimates are obtained using integration by parts rules. Therefore, we make use of the similar SBP rules for the discrete approximations of $\partial / \partial x[7]$. The sufficient condition (1.14) is then deduced directly from discrete energy estimates.

According to the scalar product (1.8) we obtain

$$
\left\langle\partial_{t} U, P^{T} H_{P} P U\right\rangle_{\Delta x}(t)=\frac{\Delta x}{2}\left\langle\partial_{t}\left(H_{P} P U_{0}\right), P U_{0}\right\rangle(t)+\Delta x \sum_{j=1}^{\infty}\left\langle\partial_{t} U_{j}, P^{T} H_{P} P U_{j}\right\rangle(t) .
$$

Since $P^{T} H_{P} P$ is a symmetric positive definite matrix and using the homogeneous boundary condition $B U_{0}(t)=0$ and thus $P U_{0}=\left(0, \Pi_{2} P U_{0}\right)^{T}$, the previous equality $(2.1)$ can be reformulated as

$$
\frac{1}{2} \partial_{t}\left\langle U, P^{T} H_{P} P U\right\rangle_{\Delta x}(t)=\frac{\Delta x}{2} \partial_{t}\left(\Pi_{2} H_{P} P U_{0}\right)(t)\left(\Pi_{2} P U_{0}\right)(t)+\Delta x \sum_{j=1}^{\infty}\left\langle\partial_{t} U_{j}, P^{T} H_{P} P U_{j}\right\rangle(t) .
$$


Now, we show how the difference operator $(\mathcal{Q} U)_{j \in \mathbb{N}}$ can be applied for the IBVP (1.11) for the homogeneous boundary condition at all gridpoints including the boundary point $j=0$

$$
\partial_{t} U_{j}(t)=\frac{1}{\varepsilon} S U_{j}(t)-\frac{1}{2 \Delta x} A\left(U_{j+1}(t)-U_{j-1}(t)\right), \quad j \geq 1,
$$

and

$$
\partial_{t}\left(\Pi_{2} H_{P} P U_{0}\right)(t)=\frac{1}{\varepsilon} \Pi_{2} H_{P} P S U_{0}(t)-\frac{1}{\Delta x} \Pi_{2} H_{P} P A\left(U_{1}-U_{0}\right)(t) .
$$

Thus, (2.2) can be represented as

$$
\begin{aligned}
\partial_{t}\left\langle U, P^{T} H_{P} P U\right\rangle_{\Delta x}(t)= & \frac{\Delta x}{\varepsilon}\left(\Pi_{2} H_{P} P S U_{0}\right)(t)\left(\Pi_{2} P U_{0}\right)(t)+\frac{2 \Delta x}{\varepsilon} \sum_{j=1}^{\infty}\left\langle S U_{j}, P^{T} H_{P} P U_{j}\right\rangle(t) \\
& +\left(\Pi_{2} H_{P} P A U_{0}\right)(t)\left(\Pi_{2} P U_{0}\right)(t)-\left(\Pi_{2} H_{P} P A U_{1}\right)(t)\left(\Pi_{2} P U_{0}\right)(t) \\
& -\sum_{j=1}^{\infty}\left\langle A U_{j+1}, P^{T} H_{P} P U_{j}\right\rangle(t)+\sum_{j=1}^{\infty}\left\langle A U_{j-1}, P^{T} H_{P} P U_{j}\right\rangle(t)
\end{aligned}
$$

We observe now that

$$
\sum_{j=1}^{\infty}\left\langle A U_{j-1}, P^{T} H_{P} P U_{j}\right\rangle(t)=\left\langle A U_{0}, P^{T} H_{P} P U_{1}\right\rangle(t)+\sum_{j=1}^{\infty}\left\langle A U_{j}, P^{T} H_{P} P U_{j+1}\right\rangle(t) .
$$

Since $H_{P} P A P^{-1}$ is symmetric and $P U_{0}=\left(0, \Pi_{2} P U_{0}\right)^{T}$, one gets

$$
\left\langle A U_{0}, P^{T} H_{P} P U_{1}\right\rangle(t)=\left(\Pi_{2} H_{P} P A U_{1}\right)(t)\left(\Pi_{2} P U_{0}\right)(t),
$$

and then

$$
\sum_{j=1}^{\infty}\left\langle A U_{j-1}, P^{T} H_{P} P U_{j}\right\rangle(t)=\left(\Pi_{2} H_{P} P A U_{1}\right)(t)\left(\Pi_{2} P U_{0}\right)(t)+\sum_{j=1}^{\infty}\left\langle A U_{j+1}, P^{T} H_{P} P U_{j}\right\rangle(t) .
$$

Substituting (2.4) into (2.3), we obtain

$$
\partial_{t}\langle U, H U\rangle_{\Delta x}(t)+2 a \frac{B_{u}}{B_{v}} u_{0}^{2}(t)+\frac{\Delta x}{\varepsilon} v_{0}^{2}(t)=-\frac{2 \Delta x}{\varepsilon} \sum_{j=1}^{\infty} v_{j}^{2}(t),
$$

where the symmetric positive definite matrix $H=B_{v}^{-2} P^{T} H_{P} P$ is simply

$$
H=\left(\begin{array}{cc}
a & 0 \\
0 & 1
\end{array}\right)
$$

In order for the energy method to work, the boundary condition has to satisfy

$$
2 a \frac{B_{u}}{B_{v}} u_{0}^{2}(t)+\frac{\Delta x}{\varepsilon} v_{0}^{2}(t) \geq c\left|U_{0}(t)\right|^{2}
$$

for some constant $c>0$ whenever $B_{u} u_{0}(t)+B_{v} v_{0}(t)=0$. This leads to the sufficient condition

$$
2 a \frac{B_{u}}{B_{v}}+\frac{\Delta x}{\varepsilon}\left(\frac{B_{u}}{B_{v}}\right)^{2}>0
$$


under which we directly get the inequality

$$
\partial_{t}\langle U, H U\rangle_{\Delta x}(t)+c\left|U_{0}\right|^{2}(t) \leq 0
$$

and thus finally

$$
\langle U, H U\rangle_{\Delta x}(T)+c \int_{0}^{T}\left|U_{0}\right|^{2}(t) \mathrm{d} t \leq\langle f, H f\rangle_{\Delta x} .
$$

More into the details, the following cases occur:

- if $B_{u} B_{v}>0$ then there exists $c \leq \frac{2 a B_{u} B_{v}}{B_{u}^{2}+B_{v}^{2}}$ such that (2.5) holds uniformly.

- if $B_{u} B_{v}<0$, consider some $\delta_{0}>-2 a B_{v} B_{u}^{-1}$. Then there exists $c\left(\delta_{0}\right)>0$ such that (2.5) holds uniformly as soon as $\Delta x \geq \delta_{0} \varepsilon$ with $c=c\left(\delta_{0}\right)$. For example, if we choose $\delta_{0}=-3 a B_{v} B_{u}^{-1}$ then there exists $c \leq-\frac{a B_{u} B_{v}}{B_{u}^{2}+B_{v}^{2}}$ such that (2.5) holds uniformly.

This ends the proof of Proposition 1.3.

Let us mention that, assuming the condition (1.12) of the main theorem to be fulfilled, the condition (1.14) is then automatically satisfied. Then, from the inequality (2.5), for any $T>0$, there exists a constant $C_{T}>0$ such that the following inequality holds

$$
\int_{0}^{T} \sum_{j \geq 0} \Delta x\left|U_{j}(t)\right|^{2} \mathrm{~d} t+\int_{0}^{T}\left|U_{0}(t)\right|^{2} \mathrm{~d} t \leq C_{T} \sum_{j \geq 0} \Delta x\left|f_{j}\right|^{2} .
$$

This will be used to prove Theorem 1.2.

\subsection{Numerical experiments}

In this section we perform some numerical experiments and observe the effective behavior (i.e. the time evolution) of the energy

$$
E(t):=\langle U(t), H U(t)\rangle_{\Delta x}
$$

according to whether or not the discrete strict dissipativity condition (1.14) is valid. We also have a look at the degenerate case when the UKC (1.5) does not hold (and thus, none of the other stability conditions). As discussed in the previous section and in the calculations of Xin and $\mathrm{Xu}$ [21], we expect to observe the decrease of the energy $E(t)$ as soon as $B_{u} B_{v}>0$. What happens in the case $B_{u} B_{v}<0$, but while the condition (1.14) still holds, is also experimented.

As main parameters for the experiments, we fix the space step $\Delta x=10^{-2}$, choose $a=4$ and let $\varepsilon$ and the boundary parameter $\left(B_{u}, B_{v}\right)$ vary. Our purpose is not to discuss the choice of a time integrator for the ODE system, let us mention that in any case we make use of the integrated solver ode45 of MATLAB (explicit variable time-step Runge-Kutta $(4,5)$ formula, the Dormand-Prince pair).

The test case we consider concerns the following data. The boundary data is the homogeneous one $b \equiv 0$. The initial data is

$$
f_{j}= \begin{cases}(0,0), & \text { if } x_{j}=0 \\ (15,10)^{T}, & \text { if } 0<x_{j} \leq 1 / 2 \\ (0,0), & \text { if } x_{j}>1 / 2\end{cases}
$$

Let us first observe that these data are compatible in the corner $(x, t)=(0,0)$ in the sense that $B f_{0}(0)=b(0)$. Moreover the choice of an initial data with support in $[0,1 / 2]$ is motivated by the property of finite speed of propagation available at the continuous side (1.1). More precisely, the exact solution we approximate has characteristic velocities \pm 2 and therefore vanishes outside some space interval $[0,0.9]$ for small times in $[0,0.2]$. 

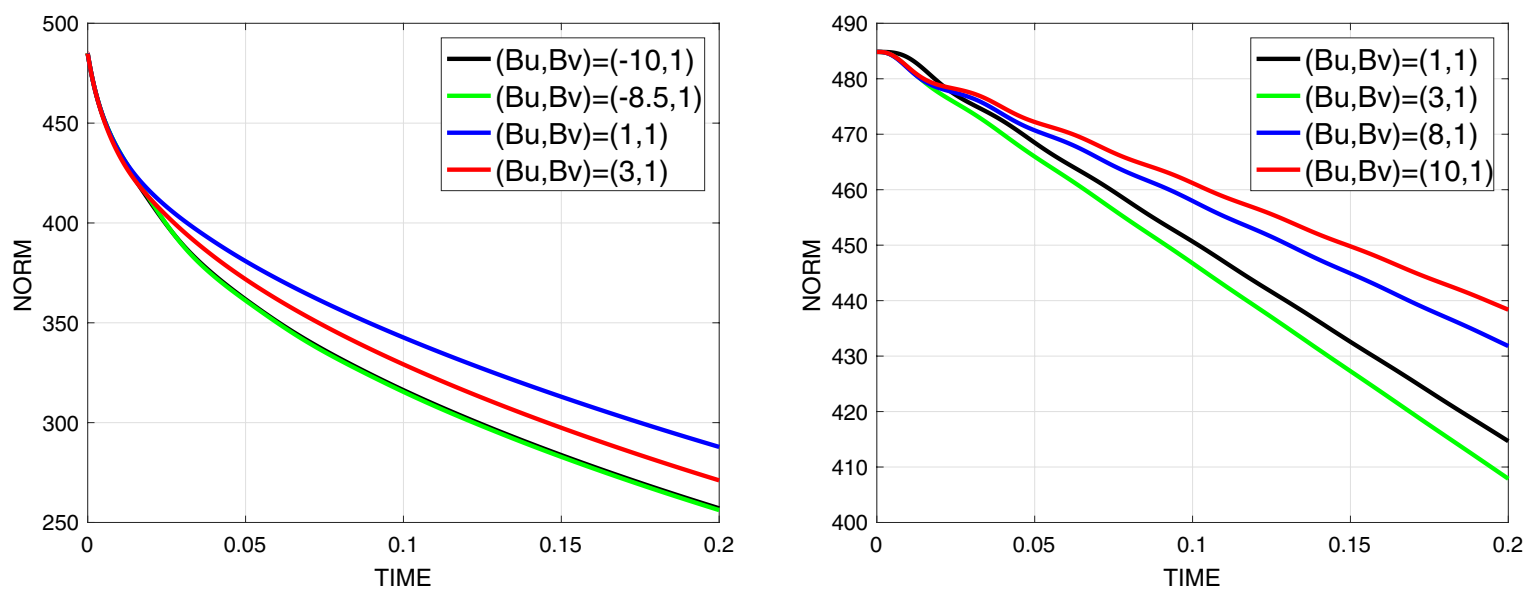

FiguRE 1. Energy evolution with condition (1.14), for $\varepsilon=10^{-2}$ (left) and $\varepsilon=10^{2}$ (right).

Thus we choose for our experiments the space interval $[0,1]$ and the time interval $[0, T]$ with $T=0.2$. Let us however mention that, strictly speaking, this analysis is actually wrong at the semi-discrete level and that in addition we have to define some discrete right boundary condition at $x=1$. The most natural choice in this situation is to select the homogeneous Neumann boundary condition $U_{J+1}(t)=U_{J}(t)$ at the rightmost cell $J$. We here don't address the precise analysis of this choice but the numerical experiments seem to behave correctly, for example when extending the space-domain to $[0,2]$. Other strategies exist in the litterature, with for example discrete transparent boundary conditions (see e.g. [2]), but we postpone these possibilities to a further work.

Firstly, we choose a set of values $\left(B_{u}, B_{v}\right)$ such that the discrete strict dissipativity condition (1.14) is satisfied with $\varepsilon=10^{-2}$ and also with $\varepsilon=10^{2}$. The Figure 1 shows the evolution of the energy $E(t)$ over the time interval $t \in[0,0.2]$.

- We proved that for any $\varepsilon \in(0,+\infty)$ and $\left(B_{u}, B_{v}\right)$ satisfying the condition $(1.14), E(t)$ is decreasing. This is strongly supported by the experiments. Observe also that the decrease of $E(t)$ is true even in the case $B_{u} B_{v}<0$ provided the condition (1.14) is true. This is the case for example for $\varepsilon=10^{-2}$ together with the parameters $\left(B_{u}, B_{v}\right)=(-8.5,1)$.

- In the case $\varepsilon=10^{-2}$ the energy $E(t)$ go down suddenly for small $t>0$. This is due to the initial relaxation of the solution to the equibrium system. In the case $\varepsilon=10^{2}$, the decrease seems to be linear. It is not so much influenced by the relaxation source term but more by the boundary dissipation.

Secondly, we choose a set of values $\left(B_{u}, B_{v}\right)$ such that the condition (1.14) is not satisfied with $\varepsilon=10^{-2}$ nor with $\varepsilon=10^{2}$. Besides, we also present the evolution of the energy for parameters such that the Uniform Kreiss Condition (1.5) is wrong. The Figure 2 shows the evolution of $E(t)$ over the time interval $t \in[0,0.2]$.

- On the boundary $x=0$, for all $\varepsilon>0$, if the boundary condition (1.3) with homogeneous boundary condition $b(t) \equiv 0$ does not satisfy the UKC, then $v^{\varepsilon}=\sqrt{a} u^{\varepsilon}$. Therefore the numerical scheme of the IBVP is not stable for each fixed $\varepsilon$. For $\varepsilon=10^{-2}$ and $\varepsilon=10^{2}$, if we choose $\left(B_{u}, B_{v}\right)=(-2,1)$ then the values of $E(t)$ increase quickly.

- When the condition (1.14) fails, then we observe for any $t \in(0,0.2]$ the inequality $E(t)>E(0)$. In the particular case $\varepsilon=10^{-2}$, the evolution is non-monotone and there exists $0<t_{1}<t_{2}$ such that $E\left(t_{1}\right)>E\left(t_{2}\right)$. However, after that the values of $E(t)$ increase rapidly. In the case $\varepsilon=10^{2}$, the values of $E(t)$ rise gradually.

Clearly, the numerical results show that the energy $E(t)$ increases in time as soon as the discrete strict dissipativity condition (1.14) does not hold. The behavior is even worse when the UKC (1.5) is not satisfied. It 

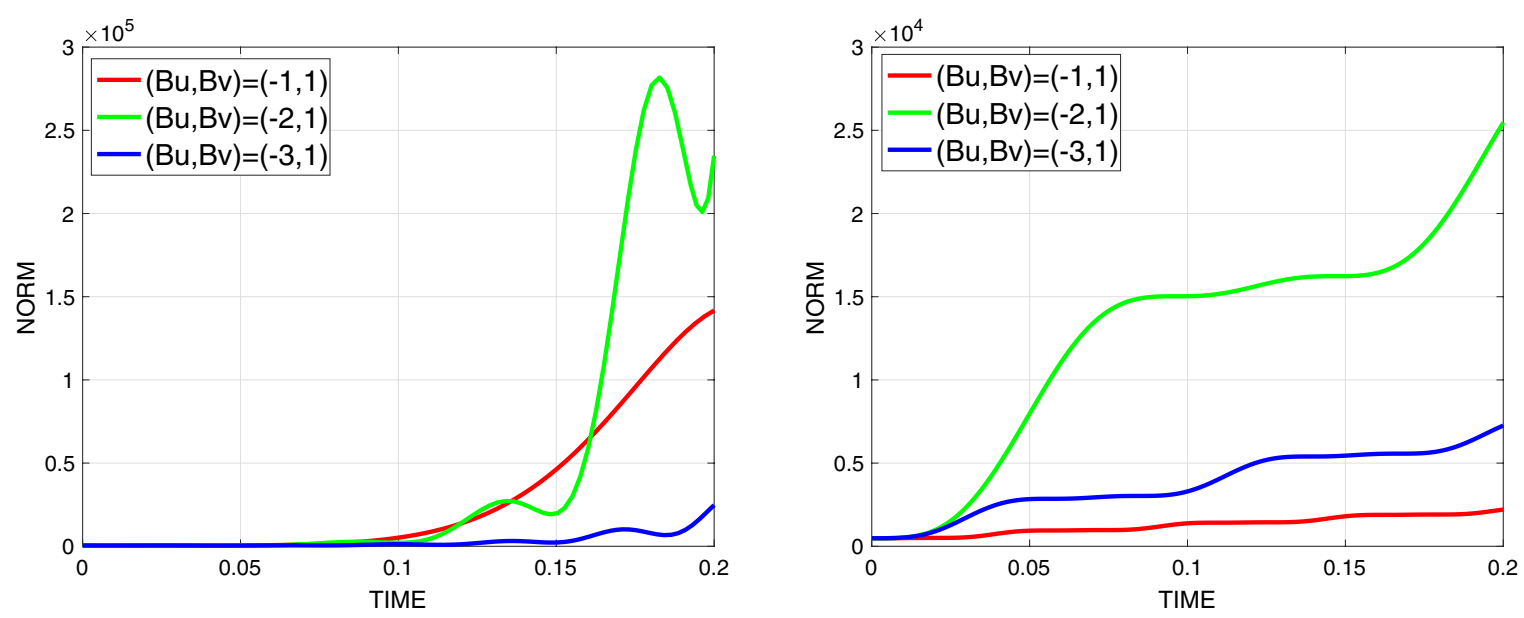

FIGURE 2. Energy evolution without condition (1.14), for $\varepsilon=10^{-2}$ (left) and $\varepsilon=10^{2}$ (right).

seems that the condition (1.14) is also necessary to ensure the non-increase of the energy, but let us stress that an increasing energy with respect to time may not be in contradiction with the stiff stability.

\section{Stiff Strong Stability of the SEMI-Discrete IBVP}

In the continuous case, the IBVP (1.1)-(1.3) is stiffly well-posed if and only if the boundary condition satisfies the SKC (1.6). Now, we want to address the question of existence of unstable solutions in order to derive a necessary condition for the stability of the discrete IBVP (1.11). Following Yong [22] and Xin and Xu [21], we shall apply the normal mode analysis to derive the strict dissipativity condition (1.12).

\subsection{Strictly dissipative boundary conditions}

We look for (nontrivial) solutions of (1.11) satisfying the homogeneous boundary condition $B U_{0}(t)=0$ and of the form

$$
U_{j}(t)=\mathrm{e}^{\xi t / \varepsilon} \phi_{j}
$$

with $\xi \in \mathbb{C}$ such that $\operatorname{Re} \xi>0$, and $\left(\phi_{j}\right)_{j \in \mathbb{N}} \in l^{2}\left(\mathbb{N}, \mathbb{C}^{2}\right)$. Such solutions, if they exist, clearly violate the $\varepsilon$-uniform $l^{2}$ estimates in (1.13). Our goal is to find a sufficient condition to ensure that they do not exist.

Substituting (3.1) into (1.11), we have to solve the following problem

$$
\begin{aligned}
\phi_{j+1}-\phi_{j-1} & =\frac{2 \Delta x}{\varepsilon} M(\xi) \phi_{j}, \quad j>0 \\
B \phi_{0} & =0, \\
\Pi_{2} P^{-T} H A\left(\phi_{1}-\left(I+\frac{\Delta x}{\varepsilon} M(\xi)\right) \phi_{0}\right) & =0,
\end{aligned}
$$

where we denote the following matrix $M(\xi)$, already used in [21]:

$$
M(\xi)=A^{-1}(S-\xi I)=\frac{1}{a}\left(\begin{array}{cc}
0 & -(1+\xi) \\
-a \xi & 0
\end{array}\right) .
$$


For convenience in the notations, we recall that the eigenvalues and eigenvectors of $M(\xi)$ can be easily found to be respectively

$$
\mu_{ \pm}(\xi)= \pm \sqrt{\frac{\xi(1+\xi)}{a}}, \quad r_{ \pm}(\xi)=\left(\begin{array}{c}
1 \\
\frac{a \mu_{\mp}(\xi)}{1+\xi}
\end{array}\right) .
$$

In the half plane $\{\xi \in \mathbb{C}: \operatorname{Re} \xi>0\}$, the complex function

$$
h(\xi)=\sqrt{\xi(1+\xi)}
$$

is analytic. (As usual, we take $\sqrt{z}$ to be the principal branch with the branch cut along the negative real axis.)

Let $\xi=\alpha+i \beta, \alpha>0$, and set $p=\alpha(1+\alpha)-\beta^{2}$ and $q=(1+2 \alpha) \beta$. Then,

$$
\operatorname{Re} h(\xi)=\operatorname{Re} \sqrt{p+i q}=\sqrt{\frac{p+\sqrt{p^{2}+q^{2}}}{2}} .
$$

Now we observe that

$$
\sqrt{p^{2}+q^{2}}=\sqrt{\left(\alpha(1+\alpha)-\beta^{2}\right)^{2}+(1+2 \alpha)^{2} \beta^{2}}=\sqrt{\left(\alpha(1+\alpha)+\beta^{2}\right)^{2}+\beta^{2}} \geq \alpha(1+\alpha)+\beta^{2},
$$

and therefore,

$$
\operatorname{Re} h(\xi) \geq \sqrt{\alpha(1+\alpha)} \geq \alpha .
$$

We further note that by using the basic inequality $\sqrt{1+x} \leq 1+x / 2$ (available for any real $x \geq-1$ ), we can also obtain a close upper bound for $\operatorname{Re} h(\xi)$

$$
\operatorname{Re} h(\xi) \leq \frac{1+2 \alpha}{2}
$$

In particular, we have from the above analysis:

$$
\operatorname{Re} \mu_{+}(\xi)>0, \quad \operatorname{Re} \mu_{-}(\xi)<0, \quad \text { for } \operatorname{Re} \xi>0 .
$$

Let $\bar{P}(\xi)$ be the $2 \times 2$ matrix whose columns are composed by the component of the vector $r_{ \pm}(\xi)$ :

$$
\bar{P}(\xi)=\left(\begin{array}{cc}
1 & 1 \\
\frac{a \mu_{-}(\xi)}{1+\xi} & \frac{a \mu_{+}(\xi)}{1+\xi}
\end{array}\right),
$$

so that $M(\xi)=\bar{P}(\xi) D(\xi) \bar{P}^{-1}(\xi)$ with $D(\xi)=\operatorname{diag}\left(\mu_{+}(\xi), \mu_{-}(\xi)\right)$. Let us also define

$$
\psi_{j}=\left(\psi_{j}^{\mathrm{I}}, \psi_{j}^{\mathrm{II}}\right)^{T}=\bar{P}^{-1}(\xi) \phi_{j}
$$

Now, the two-dimensional linear second order recurrence relations (3.2a) reads also under the form of two decoupled scalar second order linear recurrence relations

$$
\begin{aligned}
\psi_{j+1}^{\mathrm{I}}-\psi_{j-1}^{\mathrm{I}} & =\frac{2 \mu_{+}(\xi) \Delta x}{\varepsilon} \psi_{j}^{\mathrm{I}}, \\
\psi_{j+1}^{\mathrm{II}}-\psi_{j-1}^{\mathrm{II}} & =\frac{2 \mu_{-}(\xi) \Delta x}{\varepsilon} \psi_{j}^{\mathrm{II}} .
\end{aligned}
$$

Firstly, we look at the solution $\left(\psi_{j}^{I}\right)_{j \in \mathbb{N}} \in l^{2}(\mathbb{N}, \mathbb{C})$ to $(3.6 \mathrm{a})$, and assume first that the solution has the form

$$
\psi_{j}^{\mathrm{I}}=z^{j} R_{1},
$$


for some $|z|<1$ and $R_{1} \in \mathbb{C}$. Substituting the ansatz (3.7) into (3.6a), we then obtain $z$ among the values

$$
z_{ \pm}(\xi)=\frac{\mu_{+}(\xi) \Delta x}{\varepsilon} \pm \sqrt{\left(\frac{\mu_{+}(\xi) \Delta x}{\varepsilon}\right)^{2}+1} .
$$

Applying Lemma B.1 with the property $\operatorname{Re} \mu_{+}(\xi)>0$ for $\operatorname{Re} \xi>0$, we can prove

$$
\left|z_{-}(\xi)\right|=\left|\frac{-\mu_{+}(\xi) \Delta x}{\varepsilon}+\sqrt{\left(\frac{-\mu_{+}(\xi) \Delta x}{\varepsilon}\right)^{2}+1}\right|<1,
$$

while, as a consequence, $\left|z_{+}(\xi)\right|>1$. Thus, the solution in $l^{2}(\mathbb{N}, \mathbb{C})$ of $(3.6 \mathrm{a})$ can be represented as

$$
\psi_{j}^{I}=z_{-}(\xi)^{j} R_{1} .
$$

Similarly, the solution of $(3.6 \mathrm{~b})$ can be represented as

$$
\psi_{j}^{\mathrm{II}}=w_{+}(\xi)^{j} R_{2},
$$

with $R_{2} \in \mathbb{C}$ and

$$
w_{ \pm}(\xi)=\frac{\mu_{-}(\xi) \Delta x}{\varepsilon} \pm \sqrt{\left(\frac{\mu_{-}(\xi) \Delta x}{\varepsilon}\right)^{2}+1},
$$

that satisfies $\left|w_{+}(\xi)\right|<1$ by again using Lemma B.1 together with the property $\operatorname{Re} \mu_{-}(\xi)<0$ for $\operatorname{Re} \xi>0$. Again the other root satisfies $\left|w_{-}(\xi)\right|>1$.

Finally, the solution $\left(\phi_{j}\right)_{j \in \mathbb{N}} \in l^{2}\left(\mathbb{N}, \mathbb{C}^{2}\right)$ of the two-dimensional problem (3.2a) has the following form

$$
\phi_{j}=\bar{P}(\xi) Z(\xi)^{j} R
$$

with $Z(\xi)=\operatorname{diag}\left(z_{-}(\xi), w_{+}(\xi)\right)$, and some $R=\left(R_{1}, R_{2}\right)^{T} \in \mathbb{C}^{2}$ that remains undetermined at this level.

Plugging now (3.10) into the boundary conditions (3.2b) and (3.2c), $R$ has to satisfy the equations

$$
\begin{aligned}
B \bar{P} R & =0, \\
\Pi_{2} P^{-T} H A \bar{P}\left(Z(\xi)-\left(I+\frac{\Delta x}{\varepsilon} D\right)\right) R & =0 .
\end{aligned}
$$

Let us introduce the following quantities

$$
\begin{aligned}
g(\xi) & =\frac{a \mu_{+}(\xi)}{1+\xi}, \quad k(\xi)=\frac{a \mu_{-}(\xi)}{1+\xi}, \\
\delta_{1}(\xi) & =z_{-}(\xi)-\left(1+\frac{\mu_{+}(\xi) \Delta x}{\varepsilon}\right), \\
\delta_{2}(\xi) & =w_{+}(\xi)-\left(1+\frac{\mu_{-}(\xi) \Delta x}{\varepsilon}\right) .
\end{aligned}
$$

Thus, (3.11) can be reformulated simply as a linear system

$$
N(\xi) R=0
$$

where we set

$$
N(\xi)=\left(\begin{array}{cc}
B_{u}+k(\xi) B_{v} & B_{u}+g(\xi) B_{v} \\
-a \delta_{1}(\xi)\left(B_{u}-k(\xi) B_{v}\right) & -a \delta_{2}(\xi)\left(B_{u}-g(\xi) B_{v}\right)
\end{array}\right)
$$


Proposition 3.1. Assume $B_{u} B_{v}>0$ and consider some parameters $\Delta x, \varepsilon>0$. For any $\xi \in \mathbb{C}$ with $\operatorname{Re} \xi \geq 0$, one has $\operatorname{det} N(\xi) \neq 0$.

In other words, the proposition states that, under the sufficient condition $B_{u} B_{v}>0$, the scheme (1.11) with homogeneous boundary condition does not admit unstable solution of the form (3.1) in $l^{2}\left(\mathbb{N}, \mathbb{C}^{2}\right)$.

Proof. We again omit the explicit reference to $\xi$ in the notations, assuming $\operatorname{Re} \xi \geq 0$ all along this proof. From the definition (3.13) and observing $k=-g$, the quantity $\operatorname{det} N$ reads also

$$
\operatorname{det} N=a\left(\delta_{1}\left(B_{u}+g B_{v}\right)^{2}-\delta_{2}\left(B_{u}-g B_{v}\right)^{2}\right),
$$

Therefore, we have

$$
\operatorname{det} N \neq 0 \Leftrightarrow\left|1-\frac{\delta_{2}}{\delta_{1}}\left(\frac{B_{u}-g B_{v}}{B_{u}+g B_{v}}\right)^{2}\right|\left|\delta_{1}\right|\left|B_{u}+g B_{v}\right|^{2} \neq 0 .
$$

- Firstly, we prove that

$$
\left|\frac{\delta_{2}}{\delta_{1}}\left(\frac{B_{u}-g B_{v}}{B_{u}+g B_{v}}\right)^{2}\right| \neq 1
$$

Let $\delta=\Delta x / \varepsilon$, then since $\mu_{-}=-\mu_{+}$, we have

$$
\left|\frac{\delta_{2}}{\delta_{1}}\right| \leq 1 \Leftrightarrow \operatorname{Re} \sqrt{\left(\mu_{+} \delta\right)^{2}+1} \geq 0 .
$$

Furthermore, the complex function $g(\xi)$ is analytic and bounded in $\operatorname{Re} \xi \geq 0$. By the conformal mapping theorem, $g(\xi)$ maps the half plane $\operatorname{Re} \xi \geq 0$ to a simply connected closed bounded domain $\Omega \subset \mathbb{C}$ whose boundary corresponds to the image of the imaginary axis $\operatorname{Re} \xi=0$ under $g$. The boundary curve

$$
g(i \beta)=\frac{\sqrt{-a \beta^{2}+a \beta i}}{1+i \beta}, \quad-\infty \leq \beta \leq \infty
$$

is a closed curve which intersects the real axis only at $\beta=0$ and at $\beta= \pm \infty$ with $g(0)=0, g( \pm i \infty)=\sqrt{a}$. Besides, the curve is transversal to the real axis.

Since $B_{u} B_{v}>0, \operatorname{Re} g(\xi) \geq 0$ in $\operatorname{Re} \xi \geq 0$, we observe that

$$
\left|\frac{B_{u}-g B_{v}}{B_{u}+g B_{v}}\right|^{2} \leq 1 \Leftrightarrow \operatorname{Re} g \geq 0 .
$$

According to (3.15) and (3.16), we obtain

$$
\left|\frac{\delta_{2}}{\delta_{1}}\left(\frac{B_{u}-g B_{v}}{B_{u}+g B_{v}}\right)^{2}\right| \leq 1
$$

Now, we assume by contradiction that for some point $\xi$ with $\operatorname{Re} \xi \geq 0$, the following occurs

$$
\left|\frac{\delta_{2}}{\delta_{1}}\left(\frac{B_{u}-g B_{v}}{B_{u}+g B_{v}}\right)^{2}\right|=1 \Leftrightarrow\left\{\begin{array} { l } 
{ | \frac { \delta _ { 2 } } { \delta _ { 1 } } | = 1 , } \\
{ | \frac { B _ { u } - g B _ { v } } { B _ { u } + g B _ { v } } | ^ { 2 } = 1 }
\end{array} \Leftrightarrow \left\{\begin{array}{l}
\operatorname{Re} \sqrt{\left(\mu_{+} \delta\right)^{2}+1}=0, \\
\xi=0 .
\end{array}\right.\right.
$$

Since $\xi=0$, we have $\operatorname{Re} \sqrt{\left(\mu_{+} \delta\right)^{2}+1}=1$ and thus we conclude that

$$
\left|\frac{\delta_{2}}{\delta_{1}}\left(\frac{B_{u}-g B_{v}}{B_{u}+g B_{v}}\right)^{2}\right| \neq 1
$$


According to (3.17) and (3.18) we have

$$
\left|1-\frac{\delta_{2}}{\delta_{1}}\left(\frac{B_{u}-g B_{v}}{B_{u}+g B_{v}}\right)^{2}\right| \neq 0 .
$$

- Secondly, with $\delta=\Delta x / \varepsilon$, we get $\left|\delta_{1}\right|=\left|1+\sqrt{\left(\mu_{+} \delta\right)^{2}+1}\right| \geq 1$ and

$$
\left|B_{u}+g B_{v}\right|^{2} \geq B_{u}^{2}>0,
$$

due to the facts $B_{u} B_{v}>0$ and $\operatorname{Re} g(\xi) \geq 0$ for $\operatorname{Re} \xi \geq 0$.

Therefore, we proved $\operatorname{det} N \neq 0$.

\subsection{Numerical experiments for the necessity of the boundary condition}

Using the normal mode analysis, we prove that the strict dissipativity condition (1.12) is sufficient to preclude the existence of unstable solutions of the form (3.1). However, we are not able to prove that this condition is also necessary, i.e. that there exists an unstable solution to (1.11) with homogeneous boundary condition as soon as $B_{u} B_{v}<0$. We first present hereafter numerical results in this advantageous case (1.12), concerning the quantity $|\operatorname{det} N|$ introduced in (3.13). Then we also perform a numerically study for situations with $B_{u} B_{v}<0$, and more importantly when $B_{u} / B_{v}<-\sqrt{a}$, which is a sub-case of the SKC (1.6).

Let us denote the following quantity of interest, depending on $\xi \in \mathbb{C}, \delta=\Delta x / \varepsilon>0$ and the boundary parameters $\left(B_{u}, B_{v}\right)$ through their ratio:

$$
F\left(\xi, \delta, \frac{B_{u}}{B_{v}}\right)=\delta_{1}\left(\frac{B_{u}}{B_{v}}+g(\xi)\right)^{2}-\delta_{2}\left(\frac{B_{u}}{B_{v}}-g(\xi)\right)^{2},
$$

with quantities introduced in (3.12), and recall that we have

$$
\operatorname{det} N \neq 0 \Leftrightarrow F\left(\xi, \delta, \frac{B_{u}}{B_{v}}\right) \neq 0 .
$$

Our numerical study is based on two complementary methods. The first one corresponds to the display of three-dimensional data in two dimensions using contours or color-coded regions. We draw contour lines of the quantity $\left|F\left(\xi, \delta, B_{u} / B_{v}\right)\right|$ in the complex plane for $\xi$, thus computed from a grid of $\operatorname{Re} \xi$ values in the horizontal axis and a grid of $\operatorname{Im} \xi$ values in the vertical axis. For each fixed parameters $\delta$ and $B_{u} / B_{v}$, a contour line is then a curve in the $\xi$-plane along which the function $\left|F\left(\xi, \delta, B_{u} / B_{v}\right)\right|$ has a constant value, so that any curve joins points with equal values.

To know whether or not the function $F$ vanishes at some point $\xi$, which is a property that the contour lines may hardly support, we also test numerically the argument principle for the following contour integral

$$
I\left(\xi_{0}, R, \delta, B_{u} / B_{v}\right)=\frac{1}{2 \pi i} \int_{\mathcal{D}} \frac{F^{\prime}\left(\xi, \delta, B_{u} / B_{v}\right)}{F\left(\xi, \delta, B_{u} / B_{v}\right)} \mathrm{d} \xi .
$$

The involved contour curve is some positively oriented circle $\mathcal{D} \subset\{\xi \in \mathbb{C}: \operatorname{Re} \xi>0\}$ defined by

$$
\mathcal{D}=\left\{\xi \in \mathbb{C},\left|\xi-\xi_{0}\right|=R\right\}=\left\{\xi_{0}+R e^{i \theta}, \theta \in(0,2 \pi]\right\},
$$

where the parameters $\xi_{0}$ and $R>0$ are chosen by hand from the contour plots. The numerical approximation of the integral (3.19) is obtained thanks to the trapezoidal rule on a uniformly distributed grid $\xi\left(\theta_{j}\right)=\xi_{0}+$ $\operatorname{Re}^{i \theta_{j}}$, where $\theta_{j}=2 j \pi / N$ for $1 \leq j \leq N$ for some large integer $N$. This computation benefits from the wellknown spectral accuracy of the method for periodic integrand (see e.g. [19]). The numerator of (3.19) with values $F^{\prime}\left(\xi\left(\theta_{j}\right), \delta, B_{u} / B_{v}\right)$ is approximated thanks to a spectral differentiation method [18]. We thus obtain 

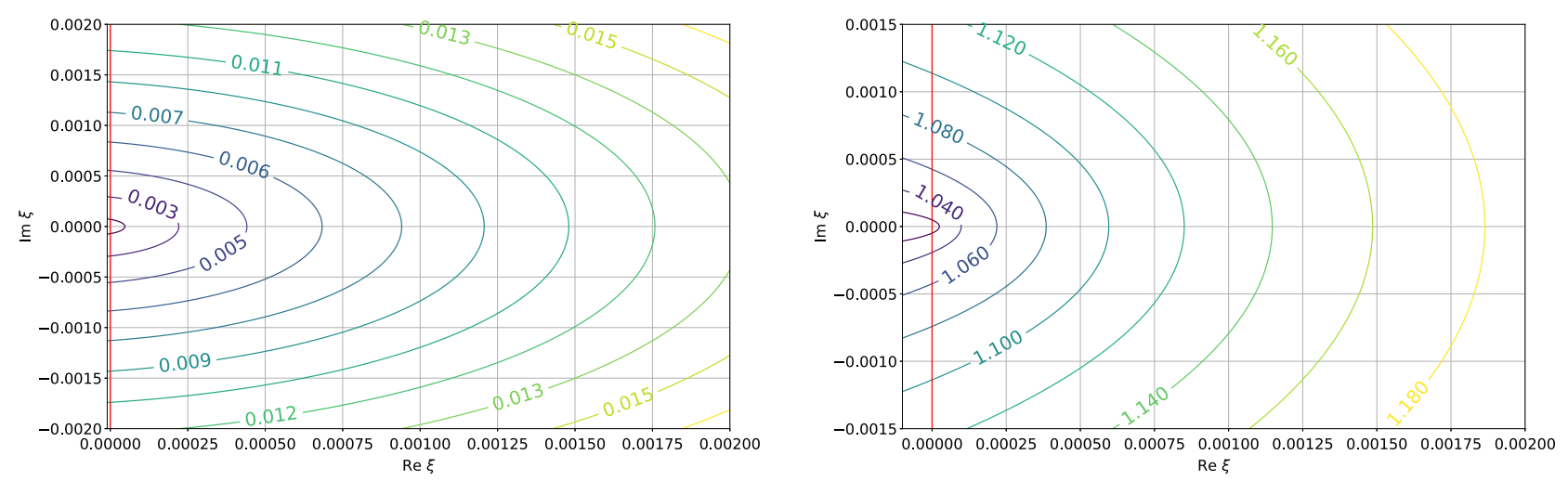

Figure 3. Contour plot of $\xi \mapsto\left|F\left(\xi, \delta, B_{u} / B_{v}\right)\right|$. The parameters are $\delta=10, B_{u} / B_{v}=1 / 40$ (left) and $\delta=10^{-2}, B_{u} / B_{v}=1$ (right).

approximations that we denote $\widehat{D F}\left(\xi\left(\theta_{j}\right), \delta, B_{u} / B_{v}\right)$. This approximation uses the discrete Fourier transform and only the pointwise evaluation of $F$ on the grid. It also has spectral accuracy for large $N$. Finally, as an approximation of $I\left(\xi_{0}, R, \delta, B_{u} / B_{v}\right)$, we consider the following quantity:

$$
I_{N}\left(\xi_{0}, R, \delta, B_{u} / B_{v}\right):=\frac{-i}{N} \sum_{j=1}^{N} \frac{\widehat{D F}\left(\xi\left(\theta_{j}\right), \delta, B_{u} / B_{v}\right)}{F\left(\xi\left(\theta_{j}\right), \delta, B_{u} / B_{v}\right)} .
$$

The function $F$ being holomorphic in the half plane $\operatorname{Re} \xi>0$, this approximation precisely counts the number of zeros (with multiplicities) inside the contour $\mathcal{D}$.

In any case, we choose $a=4$ so that SKC condition reads $B_{u} / B_{v} \notin[-2,0]$.

Firstly, for all $\xi \in \mathbb{C}$ with $\operatorname{Re} \xi>0$, we consider the values of $\left|F\left(\xi, \delta, B_{u} / B_{v}\right)\right|$ with parameters $\delta=10$ and $B_{u} / B_{v}=1 / 40>0$ (Fig. 3 left) and then with parameters $\delta=10^{-2}$ and $B_{u} / B_{v}=1>0$ (Fig. 3 right).

In both case, we observe that there exists a constant $c>0$ such that $\left|F\left(\xi, \delta, B_{u} / B_{v}\right)\right| \geq c$ in the half plane $\operatorname{Re} \xi>0$. From our experiments, the constant $c=10^{-3}$ seems suitable in the first case and $c=1$ in the second one. Actually, the above observations will be confirmed rigorously in Section 4.2.

Secondly, for all $\xi \in \mathbb{C}$ with $\operatorname{Re} \xi>0$, we consider the values of $\left|F\left(\xi, \delta, B_{u} / B_{v}\right)\right|$ with parameters $B_{u} / B_{v} \in$ $[-\sqrt{a}, 0]$ and various values for $\delta>0$. More precisely with choose $B_{u} / B_{v}=-1$ together with $\delta=1$ (Fig. 4) and $\delta=10$ (Fig. 5).

In the first case, the contour lines promote the existence of some $\xi \in \mathbb{C}$ with $\operatorname{Re} \xi>0$ satifsying $|F(\xi, 1,-1)| \ll$ 1. Therefore we consider the circled curve $\mathcal{D}$ with parameters $\xi_{0}=0.2027+0.1471 i$ and $R=2 \times 10^{-4}$. According to Table 1, we compute the contour integral and for large integers $N$, we get $I_{N}\left(\xi_{0}, R, 1,-1\right)=1$ up to the machine epsilon. Thus, there exists exactly one complex number $\xi$ inside the contour $\mathcal{D}$ such that $F(\xi, 1,-1)=0$ and a corresponding unstable solution.

In the case $\delta=10$, for any $\operatorname{Re} \xi>0$ then $|F(\xi, 10,-1)| \neq 0$ (Fig. 5). Therefore, we can not prove that for any $\delta>0, \xi \in \mathbb{C}, \operatorname{Re} \xi>0$, if $B_{u} / B_{v} \in[-\sqrt{a}, 0]$ then $\left|F\left(\xi, \delta, B_{u} / B_{v}\right)\right| \neq 0$.

Thirdly, for all $\xi \in \mathbb{C}$ with $\operatorname{Re} \xi>0$, we consider the boundary parameter $B_{u} / B_{v}=-3.5$ so that $B_{u} / B_{v}<$ $-\sqrt{a}$. Let us recall that in the continuous case, Xin and $\mathrm{Xu}$ [21] proved that there is no unstable solution in that case. Contrasting with this result, for the discrete IBVP (1.11), the next numerical experiments support the following conjecture to hold true.

Conjecture 3.2. Consider the case $B_{u} / B_{v}<-\sqrt{a}$. There exist $\delta>0$ and $\xi \in \mathbb{C}$ with $\operatorname{Re} \xi>0$ such that $\operatorname{det} N(\xi)=0$. In other words, there exists an unstable solution of (1.11) of the form (3.1). 

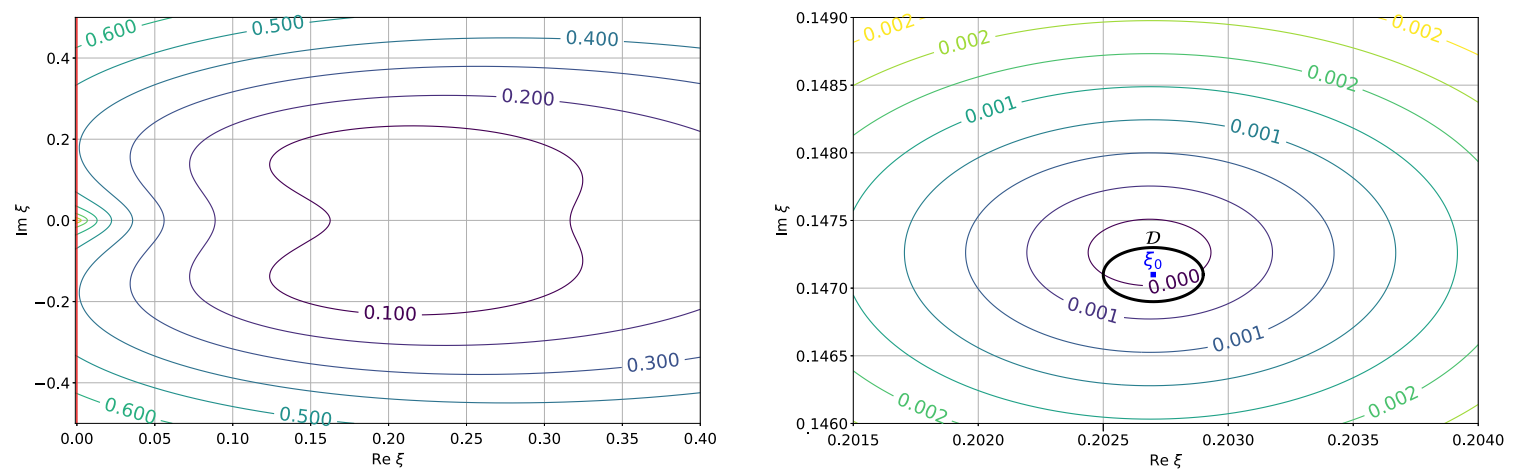

Figure 4. Contour plot of $\xi \mapsto|F(\xi, 1,-1)|$, for $\operatorname{Re} \xi>0$ (left) and a close-up near a supposed zero (right).
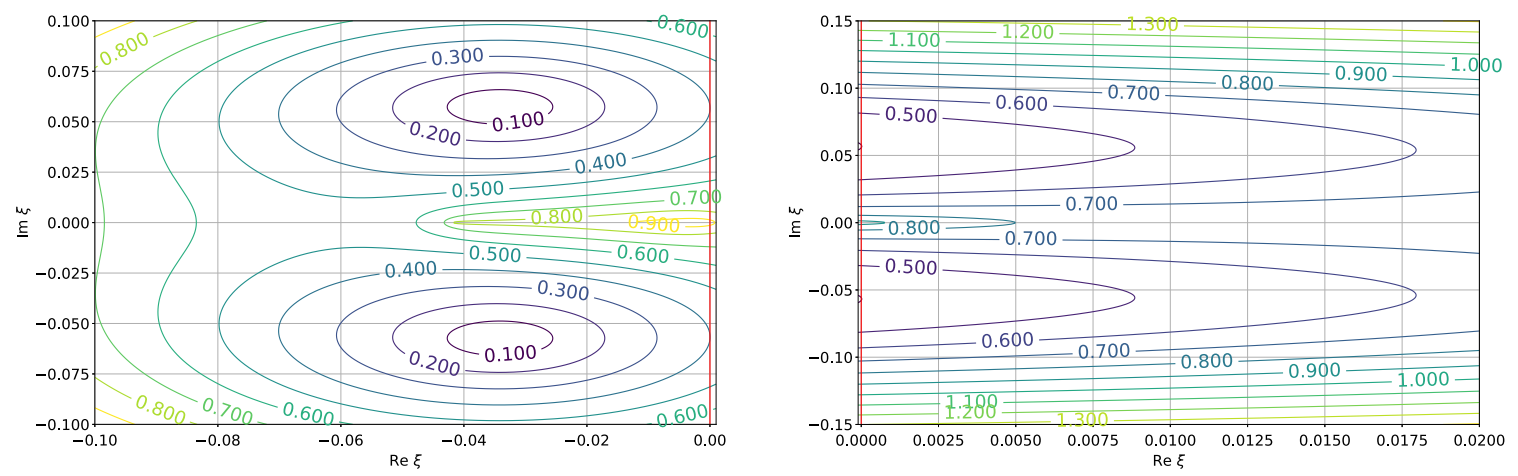

FiguRE 5. Contour plot of $\xi \mapsto|F(\xi, 10,-1)|$, for $\operatorname{Re} \xi<0$ (left) and $\operatorname{Re} \xi>0$ (right).
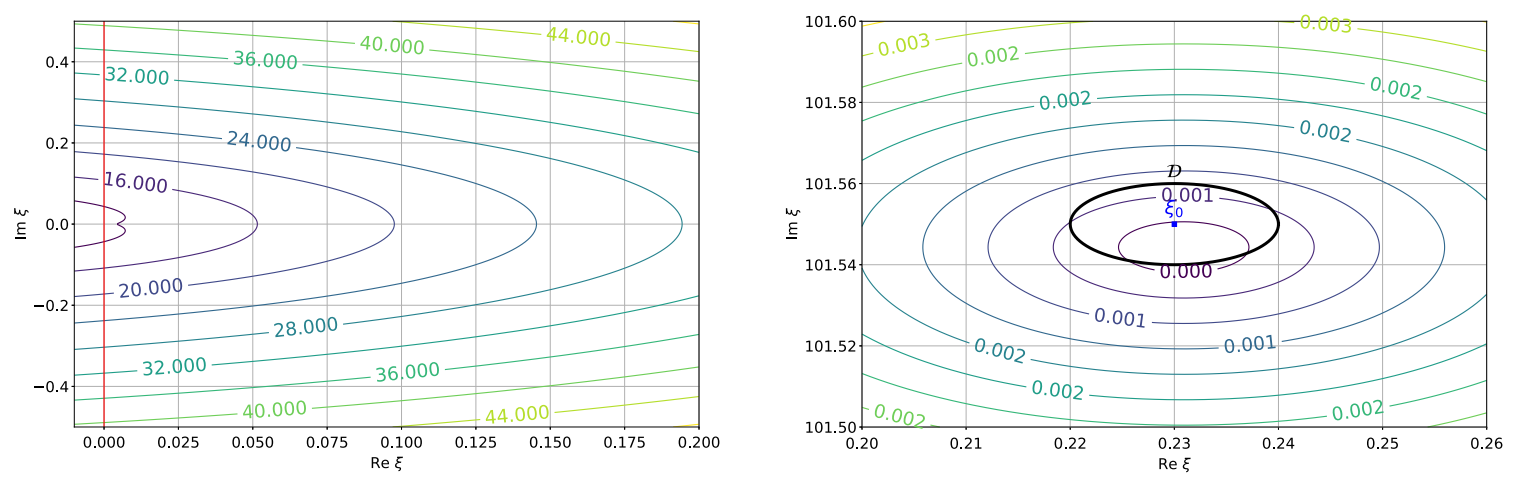

Figure 6. Contour plot of $\xi \mapsto|F(\xi, \delta,-3.5)|$ with $\delta=10$ (left) and $\delta=10^{-2}$ (right).

Now, we study the behavior of $|F(\xi, \delta,-3.5)|$ with successively $\delta=10$ and $\delta=10^{-2}$ (Fig. 6). We can see that in the case $\delta=10$, for all $\xi \in \mathbb{C}$ with $\operatorname{Re} \xi>0$, the quantity $|F(\xi, \delta,-3.5)|$ seems to be positively bounded from below. 
TABLE 1 . The contour integral $I_{N}$.

\begin{tabular}{lll}
\hline \hline$N$ & $I_{N}\left(0.2027+0.1471 i, 2 \times 10^{-4}, 1,-1\right)$ & $I_{N}\left(0.23+101.55 i, 10^{-2}, 10^{-2},-3.5\right)$ \\
\hline 20 & $0.9948572383921+0.019072730887644 i$ & $0.9999842664632257-2.3902006024 \times 10^{-8} i$ \\
40 & $0.9996520507698-0.000184801108269 i$ & $1.00000000024755+7.52237161449 \times 10^{-13} i$ \\
80 & $1.0000000869301+1.287245215 \times 10^{-7} i$ & $0.9999999999999999+4.440892098 \times 10^{-17} i$ \\
160 & $0.9999999999999+2.225615525 \times 10^{-14} i$ & $0.9999999999999999+3.747002708 \times 10^{-17} i$ \\
320 & $1.0000000000000007+1.30104 \times 10^{-17} i$ & $0.9999999999999999+1.061650767 \times 10^{-16} i$ \\
\hline
\end{tabular}

In the case $\delta=10^{-2}$ however the contour lines promote the existence of some $\xi \in \mathbb{C}$ with $\operatorname{Re} \xi>0$ satisfying $\left|F\left(\xi, 10^{-2},-3.5\right)\right| \ll 1$. Therefore we consider the circled curve $\mathcal{D}$ with parameters $\xi_{0}=0.23+101.55 i$ and $R=10^{-2}$. According to Table 1 , we compute the contour integral and for large integers $N$, we get $I_{N}\left(\xi_{0}, R, 10^{-2},-3.5\right)=1$ up to the machine epsilon. Thus, there exists a complex number $\xi$ inside the contour $\mathcal{D}$ such that $F\left(\xi, 10^{-2},-3.5\right)=0$ and a corresponding unstable solution.

\section{Stiff STABILITy of THE SEMI-DiscRete IBVP With HOMOGENEOUS INITIAL CONDITION}

For convenience in the forthcoming discussions, we recall that the semi-discrete approximation of the IBVP (1.11) with homogeneous initial condition reads

$$
\begin{cases}\partial_{t} U_{j}(t)+A \frac{U_{j+1}(t)-U_{j-1}(t)}{2 \Delta x}=\varepsilon^{-1} S U_{j}(t), & j \geq 1, t \geq 0, \\ U_{j}(0)=0, & j \geq 0, \\ B U_{0}(t)=b(t), & t \geq 0, \\ \partial_{t}\left(\Pi_{2} P^{-T} H U_{0}\right)(t)+\Pi_{2} P^{-T} H A \frac{U_{1}(t)-U_{0}(t)}{\Delta x}=\varepsilon^{-1} \Pi_{2} P^{-T} H S U_{0}(t), & t \geq 0 .\end{cases}
$$

Dealing with difference approximations, the Laplace transform is already the more powerful tool for problems in one space dimension. It is used to determine stability features when the energy method is not sufficient. Under the strict dissipativity condition (1.12), the numerical solution $\left(U_{j}(t)\right)_{j \in \mathbb{N}}$ can be constructed by the method of Laplace transform. By using the Parseval's identity, we get the expected result of Proposition 1.4.

\subsection{Solution by Laplace transform}

The numerical solution $U_{j}(t)$ of the IBVP (4.1) can be constructed by the method of Laplace transform. Let

$$
\widetilde{U}_{j}(\xi)=\mathcal{L} U_{j}=\int_{0}^{\infty} \mathrm{e}^{-\xi t} U_{j}(t) \mathrm{d} t, \quad \operatorname{Re} \xi>0 .
$$

With $U_{j}(0) \equiv 0$, we have

$$
\mathcal{L}\left(\partial_{t} U_{j}\right)=\xi \widetilde{U}_{j}(\xi)-U_{j}(0)=\xi \widetilde{U}_{j}(\xi)
$$

and therefore (4.1) become

$$
\begin{aligned}
\widetilde{U}_{j+1}(\xi)-\widetilde{U}_{j-1}(\xi) & =\frac{2 \Delta x}{\varepsilon} M(\varepsilon \xi) \widetilde{U}_{j}(\xi), \quad j>0, \\
B \widetilde{U}_{0}(\xi) & =\widetilde{b}(\xi), \\
\Pi_{2} P^{-T} H A\left(\widetilde{U}_{1}(\xi)-\left(I+\frac{\Delta x}{\varepsilon} M(\varepsilon \xi)\right) \widetilde{U}_{0}(\xi)\right) & =0,
\end{aligned}
$$


where

$$
\widetilde{b}(\xi)=\mathcal{L} b=\int_{0}^{\infty} \mathrm{e}^{-\xi t} b(t) \mathrm{d} t
$$

and the matrix $M(\varepsilon \xi)$ is the same as in (3.3).

Note that the eigenvalues $\mu_{ \pm}(\xi)$ of the matrix $M(\xi)$ satisfy

$$
\operatorname{Re} \mu_{-}(\xi)<0, \quad \operatorname{Re} \mu_{+}(\xi)>0, \quad \text { for } \operatorname{Re} \xi>0 .
$$

One can proceed as in (3.6)-(3.10) to find the solution $\widetilde{U}_{j}(\xi)$ of (4.2). For some vector $R \in \mathbb{C}^{2}$, it takes the form

$$
\widetilde{U}_{j}(\xi)=\bar{P}(\varepsilon \xi) Z^{j}(\varepsilon \xi) R .
$$

The value of $R$ can be determined easily from the boundary condition (4.3) and (4.4)

$$
R=\frac{\widetilde{b}(\xi)}{\operatorname{det} N(\varepsilon \xi)} N_{1}(\varepsilon \xi),
$$

where

$$
N_{1}(\xi)=a\left(\begin{array}{c}
-\delta_{2}(\xi)\left(B_{u}-g(\xi) B_{v}\right) \\
\delta_{1}(\xi)\left(B_{u}+g(\xi) B_{v}\right)
\end{array}\right)
$$

and the matrix $N(\varepsilon \xi)$ is the same as in (3.13). Therefore,

$$
\widetilde{U}_{j}(\xi)=\frac{\widetilde{b}(\xi)}{\operatorname{det} N(\varepsilon \xi)} \bar{P}(\varepsilon \xi) Z^{j}(\varepsilon \xi) N_{1}(\varepsilon \xi) .
$$

With $\widetilde{U}_{j}(\xi)$ found, the numerical solution $U_{j}(t)$ of (4.1) can be obtained by inverting the Laplace transform

$$
U_{j}(t)=\mathcal{L}^{-1} \widetilde{U}_{j}(\xi)=\frac{1}{2 \pi} \int_{-\infty}^{\infty} \mathrm{e}^{(\alpha+i \beta) t} \widetilde{U}_{j}(\alpha+i \beta) \mathrm{d} \beta, \quad \alpha>0 .
$$

\subsection{Stiff stability analysis}

Under the strict dissipativity condition $B_{u} B_{v}>0$, we consider Proposition 1.4 with homogeneous initial condition $\left(f_{j}\right)_{j \in \mathbb{N}} \equiv 0$ and nonzero boundary data $b(t)$. Actually, we will need a more stringent version of the estimate (3.18) uniform in $\delta>0$ and $\xi \in \mathbb{C}$ with $\operatorname{Re} \xi \geq 0$. This is the object of the next lemma.

Lemma 4.1. Assume $B_{u} B_{v}>0$. There exists $c \in(0,1)$ such that for any $\delta=\frac{\Delta x}{\varepsilon}>0$ and $\xi \in \mathbb{C}$ with $\operatorname{Re} \xi \geq 0$

$$
\left|\frac{\delta_{2}(\xi)}{\delta_{1}(\xi)}\left(\frac{B_{u}-g(\xi) B_{v}}{B_{u}+g(\xi) B_{v}}\right)^{2}\right| \leq 1-c .
$$

where $g, \delta_{1}$ and $\delta_{2}$ are defined in (3.12).

Proof. Firstly, from (3.15) and (3.16) we already observed, assuming $B_{u} B_{v}>0$, that for any $\delta>0$ and $\xi \in \mathbb{C}$ with $\operatorname{Re} \xi \geq 0$

$$
\left|\frac{\delta_{2}(\xi)}{\delta_{1}(\xi)}\right| \leq 1 \text { and }|\tau(g(\xi))| \leq 1
$$

where we denote

$$
\tau(g)=\frac{B_{u}-g B_{v}}{B_{u}+g B_{v}}
$$


Furthermore, the function $g(\xi)$ maps the half plane $\operatorname{Re} \xi \geq 0$ to a simply connected closed bounded domain $\Omega$. Thus, $|\tau(g(\xi))|$ tends to 1 only if $\operatorname{Re} g(\xi)$ goes to 0 .

Secondly, let $\xi=\alpha+i \beta$ with $\alpha \geq 0$ and $\beta \in \mathbb{R}$, after some calculations, one obtains

$$
\operatorname{Re} g(\xi)=\sqrt{\frac{a\left(p+\sqrt{p^{2}+q^{2}}\right)}{(1+\alpha)^{2}+\beta^{2}}}
$$

where

$$
p=\alpha(1+\alpha)+\beta^{2}, \quad q=\beta .
$$

Thus, for all $\alpha \geq 0, \beta \in \mathbb{R}, \operatorname{Re} g(\xi)$ goes to 0 only if $\xi$ tends to 0 . Therefore, outside a neighborhood of 0 in $\operatorname{Re} \xi \geq 0$

$$
|\tau(g(\xi))| \leq c<1
$$

Moreover, for any $\delta>0$ and $\xi \in \mathbb{C}$ with $\operatorname{Re} \xi \geq 0$, the quantity $\left|\frac{\delta_{2}(\xi)}{\delta_{1}(\xi)}\right|$ tends to 1 only if $\operatorname{Re} \sqrt{\left(\mu_{+}(\xi) \delta\right)^{2}+1}$ goes to 0 . However, in a neighborhood of 0 in $\operatorname{Re} \xi \geq 0$, for any $\delta>0, \operatorname{Re} \sqrt{\left(\mu_{+}(\xi) \delta\right)^{2}+1} \geq c_{1}>0$ (for the details, we refer the reader to the technical Lem. B.2). Thus,

$$
\left|\frac{\delta_{2}(\xi)}{\delta_{1}(\xi)}\right| \leq c_{2}<1
$$

and the result follows.

Proposition 4.2. Assume $B_{u} B_{v}>0$. There exists $C>0$ such that for any $\delta=\frac{\Delta x}{\varepsilon}>0$ and $\xi \in \mathbb{C}$ with Re $\xi \geq 0$

$$
\frac{|\operatorname{det} N(\xi)|^{2}}{\left\|N_{1}(\xi)\right\|^{2}} \geq C B_{u}^{2}
$$

Before we prove the above result, let us notice that it easily implies the previous Proposition 3.1. Actually, the reader has to understand this result as being the Uniform version of the previous one, in the same way the UKC is the uniform version of the Kreiss Condition for continuous hyperbolic PDEs, or the discrete UKC is the uniform version of the Godunov Ryabenkii condition for the (semi-)discrete IBVP, except now we also deal with the parameters $\varepsilon$ and $\Delta x$ (or equivalently with the single parameter $\delta$ ).

Proof. From (3.13) and (4.5), omitting the explicit dependence in $\xi$, the we can compute on the one hand

$$
|\operatorname{det} N|^{2}=a^{2}\left|1-\frac{\delta_{2}}{\delta_{1}}\left(\frac{B_{u}-g B_{v}}{B_{u}+g B_{v}}\right)^{2}\right|^{2}\left|\delta_{1}\right|^{2}\left|B_{u}+g B_{v}\right|^{4}
$$

and on the other hand

$$
\left\|N_{1}\right\|^{2}=a^{2}\left(1+\left|\frac{\delta_{2}}{\delta_{1}}\left(\frac{B_{u}-g B_{v}}{B_{u}+g B_{v}}\right)^{2}\right|\right)\left|\delta_{1}\right|^{2}\left|B_{u}+g B_{v}\right|^{2} .
$$

Thus we have the explicit formula

$$
\frac{|\operatorname{det} N|^{2}}{\left\|N_{1}\right\|^{2}}=\left|1-\frac{\delta_{2}}{\delta_{1}}\left(\frac{B_{u}-g B_{v}}{B_{u}+g B_{v}}\right)^{2}\right|^{2}\left(1+\left|\frac{\delta_{2}}{\delta_{1}}\left(\frac{B_{u}-g B_{v}}{B_{u}+g B_{v}}\right)^{2}\right|\right)^{-1}\left|B_{u}+g B_{v}\right|^{2} .
$$


Let us investigate separately any of the above terms. According to Lemma 4.1, there exists $c>0$ such that for any $\xi \in \mathbb{C}, \operatorname{Re} \xi \geq 0$ and $\delta>0$,

$$
\left|1-\frac{\delta_{2}}{\delta_{1}}\left(\frac{B_{u}-g B_{v}}{B_{u}+g B_{v}}\right)^{2}\right|^{2} \geq c
$$

and from (3.17), we have

$$
\left(1+\left|\frac{\delta_{2}}{\delta_{1}}\left(\frac{B_{u}-g B_{v}}{B_{u}+g B_{v}}\right)^{2}\right|\right)^{-1} \geq 1 / 2 .
$$

Since $B_{u} B_{v}>0$ and $\operatorname{Re} g(\xi) \geq 0$ for $\operatorname{Re} \xi \geq 0$, we finally get

$$
\left|B_{u}+g B_{v}\right|^{2} \geq B_{u}^{2}
$$

Therefore, there exists $C>0$ such that

$$
\frac{|\operatorname{det} N|^{2}}{\left\|N_{1}\right\|^{2}} \geq C B_{u}^{2}
$$

Now, we prove the uniform $l^{2}$ estimate (1.16). By an application of the following Parseval's identity $[7,17]$ :

$$
\int_{0}^{\infty} \mathrm{e}^{-2 \alpha t}\left|U_{j}(t)\right|^{2} \mathrm{~d} t=\frac{1}{2 \pi} \oint_{-\infty}^{\infty}\left|\widetilde{U}_{j}(\alpha+i \beta)\right|^{2} \mathrm{~d} \beta, \quad \alpha>0
$$

we have

$$
\begin{aligned}
\int_{0}^{\infty} \mathrm{e}^{-2 \alpha t}\left|U_{0}(t)\right|^{2} \mathrm{~d} t & =\frac{1}{2 \pi} \oint_{-\infty}^{\infty}\left|\widetilde{U}_{0}(\alpha+i \beta)\right|^{2} \mathrm{~d} \beta \\
& =\frac{1}{2 \pi} \oint_{-\infty}^{\infty}|\widetilde{b}(\xi)|^{2} \frac{\left\|N_{1}(\varepsilon \xi)\right\|^{2}}{|\operatorname{det} N(\varepsilon \xi)|^{2}}|\bar{P}(\varepsilon \xi)|^{2} \mathrm{~d} \beta
\end{aligned}
$$

where $\xi=\alpha+i \beta$. We fix $\alpha>0$ from now on.

According to Proposition 4.2, there exists $C_{1}>0$ such that for any $\delta>0, \xi \in \mathbb{C}, \operatorname{Re} \xi \geq 0$,

$$
\frac{\left\|N_{1}(\varepsilon \xi)\right\|^{2}}{|\operatorname{det} N(\varepsilon \xi)|^{2}} \leq C_{1} \text {. }
$$

On the other hand, since $k(\xi)=-g(\xi)$ is uniformly bounded in $\operatorname{Re} \xi \geq 0$, we obtain

$$
\begin{aligned}
\int_{0}^{\infty} \mathrm{e}^{-2 \alpha t}\left|U_{0}(t)\right|^{2} \mathrm{~d} t & \lesssim \frac{1}{2 \pi} \oint_{-\infty}^{\infty}|\widetilde{b}(\alpha+i \beta)|^{2} \mathrm{~d} \beta \\
& \lesssim \int_{0}^{\infty} \mathrm{e}^{-2 \alpha t}|b(t)|^{2} \mathrm{~d} t .
\end{aligned}
$$

This, together with a consequence of the hyperbolicity of (1.1) by using the classical argument of changing the data $b$ to zero after time $T$ and unchanged before time $T$, we obtain the desired boundary estimate

$$
\int_{0}^{T}\left|U_{0}(t)\right|^{2} \mathrm{~d} t \leq K_{T} \int_{0}^{T}|b(t)|^{2} \mathrm{~d} t .
$$

Similarly, by an application of the Parseval's identity, we have 


$$
\begin{aligned}
\int_{0}^{\infty} \sum_{j \geq 0} \mathrm{e}^{-2 \alpha t}\left|U_{j}(t)\right|^{2} \mathrm{~d} t & =\frac{1}{2 \pi} \oint_{-\infty}^{\infty} \sum_{j \geq 0}\left|\widetilde{U}_{j}(\alpha+i \beta)\right|^{2} \mathrm{~d} \beta \\
& =\frac{1}{2 \pi} \oint_{-\infty}^{\infty} \sum_{j \geq 0}|\widetilde{b}(\xi)|^{2} \frac{\left\|N_{1}(\varepsilon \xi)\right\|^{2}}{\mid \operatorname{det} N(\varepsilon \xi))\left.\right|^{2}}|\bar{P}(\varepsilon \xi)|^{2}\left(\left|z_{-}(\varepsilon \xi)\right|^{2 j}+\left|w_{+}(\varepsilon \xi)\right|^{2 j}\right) \mathrm{d} \beta
\end{aligned}
$$

where $z_{-}(\varepsilon \xi)$ and $w_{+}(\varepsilon \xi)$ are the same as in (3.8) and (3.9).

Since $k(\varepsilon \xi)=-g(\varepsilon \xi)$ is uniformly bounded in $\operatorname{Re} \xi \geq 0, \varepsilon>0$ and using Proposition 4.2, we obtain

$$
\int_{0}^{\infty} \sum_{j \geq 0} \mathrm{e}^{-2 \alpha t}\left|U_{j}(t)\right|^{2} \mathrm{~d} t \lesssim \frac{1}{2 \pi} \oint_{-\infty}^{\infty} \sum_{j \geq 0}|\widetilde{b}(\xi)|^{2}\left(\left|z_{-}(\varepsilon \xi)\right|^{2 j}+\left|w_{+}(\varepsilon \xi)\right|^{2 j}\right) \mathrm{d} \beta .
$$

On the other hand, since $\mu_{-}(\xi)=-\mu_{+}(\xi)$ in $\operatorname{Re} \xi \geq 0$, we get $\left|z_{-}(\varepsilon \xi)\right|=\left|w_{+}(\varepsilon \xi)\right|$, and thus

$$
\int_{0}^{\infty} \sum_{j \geq 0} \mathrm{e}^{-2 \alpha t}\left|U_{j}(t)\right|^{2} \mathrm{~d} t \lesssim \frac{1}{2 \pi} \oint_{-\infty}^{\infty} \sum_{j \geq 0}\left|w_{+}(\varepsilon \xi)\right|^{2 j}|\widetilde{b}(\xi)|^{2} \mathrm{~d} \beta
$$

According to (3.4) and (3.5), for all $\varepsilon>0, \xi \in \mathbb{C}, \operatorname{Re} \xi>0$, we have

$$
\operatorname{Re} \mu_{-}(\varepsilon \xi) \leq-\frac{\varepsilon \operatorname{Re} \xi}{\sqrt{a}}<0
$$

Furthermore, we can prove

$$
\left(\frac{\operatorname{Re} \mu_{-}(\varepsilon \xi) \Delta x}{\varepsilon}+\sqrt{\left(\frac{\operatorname{Re} \mu_{-}(\varepsilon \xi) \Delta x}{\varepsilon}\right)^{2}+1}\right)^{2} \leq\left(\eta \Delta x+\sqrt{\eta^{2} \Delta x^{2}+1}\right)^{2},
$$

where $\eta=-\frac{\operatorname{Re} \xi}{\sqrt{a}}$. According to Lemma B.1 and (4.9), we have now

$$
\sum_{j \geq 0}\left|w_{+}(\varepsilon \xi)\right|^{2 j}=\left(1-\left|\frac{\mu_{-}(\varepsilon \xi) \Delta x}{\varepsilon}+\sqrt{\left(\frac{\mu_{-}(\varepsilon \xi) \Delta x}{\varepsilon}\right)^{2}+1}\right|^{2} \leq\left(1-\left(\eta \Delta x+\sqrt{\eta^{2} \Delta x^{2}+1}\right)^{2}\right)^{-1} .\right.
$$

If we assume that $\Delta x \leq-\frac{3}{4 \eta}$ then $\sum_{j \geq 0}\left|w_{+}(\varepsilon \xi)\right|^{2 j} \leq-\eta^{-1} \Delta x^{-1}$, and therefore, by an application of the Parseval's identity

$$
\alpha \Delta x \int_{0}^{\infty} \sum_{j \geq 0} \mathrm{e}^{-2 \alpha t}\left|U_{j}(t)\right|^{2} \mathrm{~d} t \lesssim \frac{1}{2 \pi} \oint_{-\infty}^{\infty}|\widetilde{b}(\xi)|^{2} \mathrm{~d} \beta \lesssim \int_{0}^{\infty} \mathrm{e}^{-2 \alpha t}|b(t)|^{2} \mathrm{~d} t .
$$

According to (4.7) and (4.10), there exists $c>0$ such that

$$
\alpha \Delta x \int_{0}^{\infty} \sum_{j \geq 0} \mathrm{e}^{-2 \alpha t}\left|U_{j}(t)\right|^{2} \mathrm{~d} t+\int_{0}^{\infty} \mathrm{e}^{-2 \alpha t}\left|U_{0}(t)\right|^{2} \mathrm{~d} t \leq c \int_{0}^{\infty} \mathrm{e}^{-2 \alpha t}|b(t)|^{2} \mathrm{~d} t .
$$

This ends the proof of Proposition 1.4. 
To complete the proof of Theorem 1.2, observe that from (4.8), (4.10) and the hyperbolicity of (1.1), for any $T>0$, there exists a constant $C_{T}>0$ such that

$$
\int_{0}^{T} \sum_{j \geq 0} \Delta x\left|U_{j}(t)\right|^{2} \mathrm{~d} t+\int_{0}^{T}\left|U_{0}(t)\right|^{2} \mathrm{~d} t \leq C_{T} \int_{0}^{T}|b(t)|^{2} \mathrm{~d} t .
$$

By linearity, we can break up the IBVP (1.11) into two simpler problems, one with homogeneous initial condition and the other with homogeneous boundary condition. Finally from (2.6) and (4.12), we get the expected result of Theorem 1.2.

\section{Appendix A. Modeling An Elastic String}

\section{A.1. Derivation of the damped wave equation}

The damped wave equation in one space dimension can be derived in a variety of different physical settings. As an example of how waves occur in physical systems, we now derive the damped wave equation for a stretched string. Other physical systems, such as sound waves in air, can be analyzed in a similar way (see $[6,14])$. We start by considering model the action of an elastic string over time. Consider a tiny element of the string between $x$ and $x+\Delta x$

The following quantities are needed in our derivation (see Fig. A.1):

- $w(x, t)$ denotes vertical displacement of the string from the $x$-axis at position $x$ and time $t$.

- $\theta(x, t)$ is an angle between the string and a horizontal line at position $x$ and time $t$.

$-T(x, t)$ is a tension in the string at position $x$.

We can dispose of all the $\theta$ 's observing from the figure that

$$
\tan \theta(x, t)=\lim _{\Delta x \rightarrow 0} \frac{\Delta w}{\Delta x}=\frac{\partial w}{\partial x}: \text { slope of tangent at }(x, t) \text { in } w x \text {-plane. }
$$

The Newton's Second Law of Motion $(F=m a)$ states that

$$
F=(\rho \Delta x) \frac{\partial^{2} w}{\partial t^{2}}
$$

where $\rho$ is the linear density of the string and $\Delta x$ is the length of the segment.

The force $F$ comes from the tension in the string and also the damping force. The damping force acts in the opposite direction to the motion and is denoted by $-c \frac{\partial w}{\partial t}$ with $c>0$. We assume for our model that there are

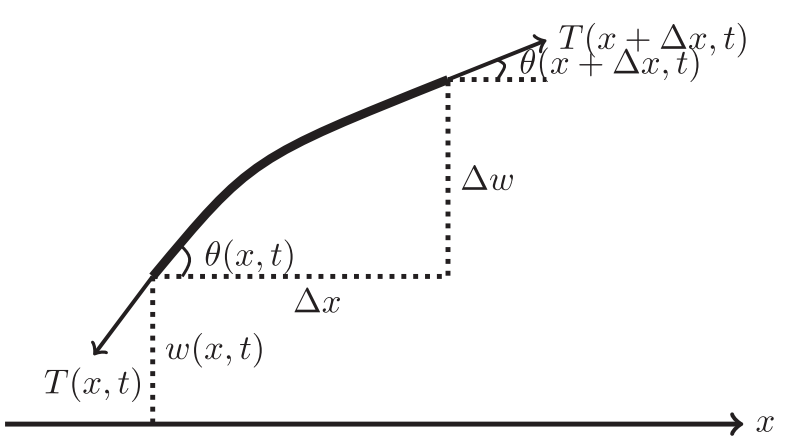

Figure A.1. Modeling an elastic string. 
only transverse vibrations, and so the string does not move horizontally, but only vertically. So, we know that the total horizontal force must be zero. Balancing the forces in the horizontal direction gives

$$
T(x+\Delta x, t) \cos \theta(x+\Delta x, t)=T(x, t) \cos \theta(x, t)=\tau,
$$

where $\tau$ is the constant horizontal tension. Balancing the forces in the vertical direction yields

$$
\begin{aligned}
F & =T(x+\Delta x, t) \sin \theta(x+\Delta x, t)-T(x, t) \sin \theta(x, t)-c \frac{\partial w}{\partial t} \Delta x \\
& =T(x+\Delta x, t) \cos \theta(x+\Delta x, t) \tan \theta(x+\Delta x, t)-T(x, t) \cos \theta(x, t) \tan \theta(x, t)-c \frac{\partial w}{\partial t} \Delta x
\end{aligned}
$$

Substituting (A.3) into (A.4) yields,

$$
F=\tau(\tan \theta(x+\Delta x, t)-\tan \theta(x, t))-c \frac{\partial w}{\partial t} \Delta x=\tau\left(\frac{\partial w}{\partial x}(x+\Delta x, t)-\frac{\partial w}{\partial x}(x, t)\right)-c \frac{\partial w}{\partial t} \Delta x .
$$

So, the vertical component of Newton's Law becomes

$$
\rho \frac{\partial^{2} w}{\partial t^{2}}(\xi, t)=\tau \frac{1}{\Delta x}\left(\frac{\partial w}{\partial x}(x+\Delta x, t)-\frac{\partial w}{\partial x}(x, t)\right)-c \frac{\partial w}{\partial t}
$$

for $\xi \in[x, x+\Delta x]$. Dividing by $\rho$ and letting $\Delta x$ tends to 0 gives

$$
\frac{\partial^{2} w}{\partial t^{2}}=\frac{\tau}{\rho} \frac{\partial^{2} w}{\partial x^{2}}-\frac{c}{\rho} \frac{\partial w}{\partial t}
$$

In order to guarantee that the equation (A.5) has a unique solution, some initial and boundary conditions have to be suitably selected: two initial conditions and boundary condition (see $[6,14]$ ).

\section{A.2. Initial conditions}

The initial position of the string and its initial velocity may be written as follow

$$
w(x, 0)=f(x) \quad \text { and } \quad w_{t}(x, 0)=h(x) .
$$

To see why we need two initial condition, note that the Taylor series of $w(x, t)$ about $t=0$ is

$$
w(x, t)=w(x, 0)+w_{t}(x, 0) t+w_{t t}(x, 0) \frac{t^{2}}{2}+w_{t t t}(x, 0) \frac{t^{3}}{3 !}+\ldots
$$

From the initial condition (A.6) and the PDE (A.5) give

$$
\begin{aligned}
w_{t t}(x, 0) & =(\tau / \rho) w_{x x}(x, 0)-(c / \rho) w_{t}(x, 0)=(\tau / \rho) f^{\prime \prime}(x)-(c / \rho) h(x), \\
w_{t t t}(x, 0) & =(\tau / \rho) w_{t x x}(x, 0)-(c / \rho) w_{t t}(x, 0)=(\tau / \rho) h^{\prime \prime}(x)-\left(c \tau / \rho^{2}\right) f^{\prime \prime}(x)+(c / \rho)^{2} h(x) .
\end{aligned}
$$

Higher order terms can be found similarly. Therefore, the two initial conditions for $w(x, 0)$ and $w_{t}(x, 0)$ are sufficient to determine $w(x, t)$ near $t=0$.

\section{A.3. Boundary condition}

We assumed the string is connected to frictionless cylinders of mass $m_{1}$ that move vertically on tracks at $x=0$ with an acceleration $g(t)$. 


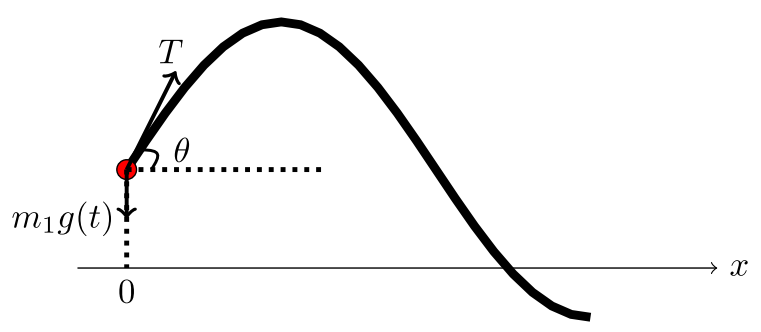

Figure A.2. Boundary condition at $x=0$.

Lemma A.1. For any $c>0, \rho>0$ and $m_{1}>0$, the boundary condition can be rewritten as follows

$$
B_{u} w_{x}(0, t)-B_{v} w_{t}(0, t)=g(t) .
$$

with $B_{u} B_{v}>0$.

Proof. Performing the force balance at $x=0$ gives

$$
T \sin \theta-c \frac{\partial w}{\partial t}=m_{1} g(t)
$$

In other words, the vertical tension in the string balances the mass of the cylinder. However, $\tau=T \cos \theta=$ const and $\tan \theta=w_{x}$, so that the previous equation becomes

$$
T \cos \theta \tan \theta-c \frac{\partial w}{\partial t}=m_{1} g(t)
$$

or also, denoting $B_{u}=\tau / m_{1}$ and $B_{v}=c / m_{1}$

$$
B_{u} w_{x}(0, t)-B_{v} w_{t}(0, t)=g(t) .
$$

To summarize, the IBVP of the linear damped wave equation in one space dimension reads (see Fig. A.2)

$$
\begin{array}{cl}
\mathrm{PDE}: \frac{\partial^{2} w}{\partial t^{2}}(x, t)=a \frac{\partial^{2} w}{\partial x^{2}}(x, t)-\frac{1}{\varepsilon} \frac{\partial w}{\partial t}(x, t), & x>0, t>0, \\
\mathrm{BC}: B_{u} w_{x}(0, t)-B_{v} w_{t}(0, t)=g(t), & t>0, \\
\mathrm{IC}: w(x, 0)=f(x), \quad w_{t}(x, 0)=h(x) & t>0 .
\end{array}
$$

where $a=\tau / \rho$, and $\varepsilon=\rho / c$. Let now denote $u^{\varepsilon}(x, t)=w_{x}(x, t)$ and $v^{\varepsilon}(x, t)=-w_{t}(x, t)$. The previous IBVP can be represented as

$$
\begin{aligned}
\partial_{t} u^{\varepsilon}(x, t)+\partial_{x} v^{\varepsilon}(x, t) & =0 \\
\partial_{t} v^{\varepsilon}(x, t)+a \partial_{x} u^{\varepsilon}(x, t) & =-\frac{1}{\varepsilon} v^{\varepsilon}(x, t),
\end{aligned}
$$

with the initial data

$$
u^{\varepsilon}(x, 0)=f^{\prime}(x), \quad v^{\varepsilon}(x, 0)=-h(x),
$$

and the linear boundary condition

$$
B_{u} u^{\varepsilon}(0, t)+B_{v} v^{\varepsilon}(0, t)=g(t)
$$

Remark A.2. The boundary condition $B_{u} B_{v}>0$ corresponds to stability condition (1.12) of the linear damped wave equation (A.7). 


\section{Appendix B. TeChnical Lemmas}

Lemma B.1. Let $v \in \mathbb{C}$ with $\operatorname{Re} v<0$, then $\left|v+\sqrt{v^{2}+1}\right| \leq \operatorname{Re} v+\sqrt{(\operatorname{Re} v)^{2}+1}<1$.

Proof. Assume that $v=x+y i$ with $x<0$ and $y \in \mathbb{R}$.

Case 1. Consider first the easy case $y=0$. Then

$$
\left|v+\sqrt{v^{2}+1}\right|=x+\sqrt{x^{2}+1},
$$

but since $x<0$, one obtains by simple considerations the inequality $x+\sqrt{x^{2}+1}<1$.

Case 2. In the general case $y \neq 0$, let us begin with some notations:

$$
\begin{gathered}
v^{2}+1=p_{1}+q_{1} i, \quad \text { with } p_{1}=x^{2}-y^{2}+1 \text { and } q_{1}=2 x y, \\
\sqrt{v^{2}+1}=a_{1}+b_{1} i, \quad \text { with } a_{1}=\sqrt{\frac{p_{1}+\sqrt{p_{1}^{2}+q_{1}^{2}}}{2}}, \text { and } b_{1}=\operatorname{sgn}\left(q_{1}\right) \sqrt{\frac{-p_{1}+\sqrt{p_{1}^{2}+q_{1}^{2}}}{2}} .
\end{gathered}
$$

Together with these notations, some algebraic identities are available:

$$
x^{2}+b_{1}^{2}+1=a_{1}^{2}+y^{2} \quad \text { and } \quad y=\frac{a_{1} b_{1}}{x} .
$$

Firstly, we prove the next inequality

$$
a_{1} x^{2}+b_{1}^{2} x+a_{1} b_{1}^{2} \geq 0 .
$$

We can see that the inequality (B.2) is equivalent to $a_{1}\left(x^{2}+b_{1}^{2}\right) \geq-x b_{1}^{2}$ and since $x<0$, the latter is now equivalent to its squared version, that reads

$$
a_{1}^{2} x^{2}\left(x^{2}+2 b_{1}^{2}\right) \geq b_{1}^{4}\left(x^{2}-a_{1}^{2}\right) .
$$

By the definition of $a_{1}, b_{1}$ above, the previous inequality is successively

$$
\begin{aligned}
& 4 x^{2}\left(p_{1}+\sqrt{p_{1}^{2}+q_{1}^{2}}\right)\left(x^{2}-p_{1}+\sqrt{p_{1}^{2}+q_{1}^{2}}\right) \geq\left(-p_{1}+\sqrt{p_{1}^{2}+q_{1}^{2}}\right)^{2}\left(2 x^{2}-p_{1}-\sqrt{p_{1}^{2}+q_{1}^{2}}\right) \\
\Leftrightarrow & 4 x^{4}\left(p_{1}+\sqrt{p_{1}^{2}+q_{1}^{2}}\right)+2 x^{2} q_{1}^{2} \geq\left(p_{1}-\sqrt{p_{1}^{2}+q_{1}^{2}}\right)\left(4 x^{2} p_{1}+q_{1}^{2}\right) \\
\Leftrightarrow & 4 x^{4}\left(p_{1}+\sqrt{p_{1}^{2}+q_{1}^{2}}\right)+2 x^{2} \times 4 x^{2} y^{2} \geq\left(p_{1}-\sqrt{p_{1}^{2}+q_{1}^{2}}\right)\left(4 x^{2}\left(x^{2}-y^{2}+1\right)+4 x^{2} y^{2}\right) \\
\Leftrightarrow & 2 x^{2}\left(\sqrt{p_{1}^{2}+q_{1}^{2}}+y^{2}\right) \geq p_{1}-\sqrt{p_{1}^{2}+q_{1}^{2}} .
\end{aligned}
$$

But, for any $p_{1}, q_{1} \in \mathbb{R}$, this is easy to see that $p_{1}-\sqrt{p_{1}^{2}+q_{1}^{2}} \leq 0$ and thus any of the previous inequalities and so the expected one (B.2) follow.

Now let us observe that the required inequality $\left|v+\sqrt{v^{2}+1}\right| \leq x+\sqrt{x^{2}+1}$ is fully equivalent to

$$
\left(x+a_{1}\right)^{2}+\left(y+b_{1}\right)^{2} \leq\left(x+\sqrt{x^{2}+1}\right)^{2},
$$

that we prove now. According to the algebraic identities in (B.1), by eliminating the occurences of $y$, the previous formula is equivalent to

$$
a_{1} x+b_{1}^{2}+\frac{a_{1} b_{1}^{2}}{x} \leq x \sqrt{x^{2}+1} .
$$


In addition, we observe that $x^{2}+1=x^{-2}\left(a_{1}^{2} x^{2}+a_{1}^{2} b_{1}^{2}-b_{1}^{2} x^{2}\right)$, and thus the previous inequality is equivalent to

$$
a_{1} x+b_{1}^{2}+\frac{a_{1} b_{1}^{2}}{x} \leq-\sqrt{a_{1}^{2} x^{2}+a_{1}^{2} b_{1}^{2}-b_{1}^{2} x^{2}}
$$

Since $x<0$ and from the inequality (B.2), the formula (B.4) reads also

$$
\begin{aligned}
& \left(a_{1} x^{2}+b_{1}^{2} x+a_{1} b_{1}^{2}\right)^{2} \geq x^{2}\left(a_{1}^{2} x^{2}+a_{1}^{2} b_{1}^{2}-b_{1}^{2} x^{2}\right) \\
\Leftrightarrow & \left(x+a_{1}\right)^{2}\left(x^{2}+b_{1}^{2}\right) \geq 0 .
\end{aligned}
$$

This ends the proof of the inequality (B.3). Now since $\operatorname{Re} v<0$, the analysis of the first easy case again applies to get $\operatorname{Re} v+\sqrt{(\operatorname{Re} v)^{2}+1}<1$.

This ends the proof of Lemma B.1.

Lemma B.2. Let $a>0$ be fixed and consider for any $\xi \in \mathbb{C}$ with $\operatorname{Re} \xi \in[0,1]$ and $\operatorname{Im} \xi \in[-1,1]$ :

$$
\mu_{+}(\xi)=\sqrt{\frac{\xi(1+\xi)}{a}} .
$$

There exists a constant $c>0$, independent of $\delta$ and $\xi$ such that

$$
\operatorname{Re} \sqrt{1+\left(\mu_{+}(\xi) \delta\right)^{2}} \geq c
$$

Proof. Let us denote $\xi=\alpha+i \beta$ with $\alpha \in[0,1]$ and $\beta \in[-1,1]$ and introduce the notation $\bar{\delta}=\delta^{2} / a$. After some calculations, one obtains

$$
\operatorname{Re} \sqrt{1+\left(\mu_{+}(\xi) \delta\right)^{2}}=\frac{1}{\sqrt{2}} \sqrt{h(\alpha, \beta, \bar{\delta})}
$$

with the function with positive real values:

$$
h(\alpha, \beta, \bar{\delta})=1+\bar{\delta}\left(\alpha(1+\alpha)-\beta^{2}\right)+\sqrt{\left(1+\bar{\delta}\left(\alpha(1+\alpha)-\beta^{2}\right)\right)^{2}+\bar{\delta}^{2} \beta^{2}(1+2 \alpha)^{2}} .
$$

Now, the required uniform lower bound will be provided directly by a uniform lower bound for the quantity $h(\alpha, \beta, \bar{\delta})$, what we are looking for now by exhaustion.

Case 1. For any $\bar{\delta}>0, \alpha \in[0,1]$ and $\beta=0$, we have the simple lower bound

$$
h(\alpha, 0, \bar{\delta})=2(1+\bar{\delta} \alpha(1+\alpha)) \geq 2 .
$$

Case 2. For any $\bar{\delta}>0, \alpha \in[0,1]$ and $\beta \in[-1,0) \cup(0,1]$ such that $\beta^{2} \leq \alpha(1+\alpha)$, we get

$$
h(\alpha, \beta, \bar{\delta}) \geq 1+\sqrt{1+\bar{\delta}^{2} \beta^{2}(1+2 \alpha)^{2}} \geq 2 .
$$

Case 3. For any $\bar{\delta}>0, \alpha \in[0,1]$ and $\beta \in[-1,0) \cup(0,1]$ such that $\beta^{2}>\alpha(1+\alpha)$, let us introduce the quantity $\tau:=\beta^{2}-\alpha(1+\alpha)$, so that

$$
h(\alpha, \beta, \bar{\delta})=1-\tau \bar{\delta}+\sqrt{(1-\tau \bar{\delta})^{2}+\bar{\delta}^{2} \beta^{2}(1+2 \alpha)^{2}} .
$$

Note that $\alpha$ being nonnegative

$$
0<\tau \leq \beta^{2} \leq 1
$$


Subcase 3.a. Assume that $\bar{\delta} \leq \tau^{-1}$. Then, $1-\tau \bar{\delta} \geq 0$ and therefore

$$
h(\alpha, \beta, \bar{\delta}) \geq \sqrt{(1-\tau \bar{\delta})^{2}+\bar{\delta}^{2} \beta^{2}(1+2 \alpha)^{2}} .
$$

We then can compute

$$
h^{2}(\alpha, \beta, \bar{\delta}) \geq(1-\tau \bar{\delta})^{2}+\bar{\delta}^{2} \beta^{2}(1+2 \alpha)^{2} \geq(1-\tau \bar{\delta})^{2}+\bar{\delta}^{2} \beta^{2} .
$$

From (B.5), we then have successively

$$
h^{2}(\alpha, \beta, \bar{\delta}) \geq 1-2 \tau \bar{\delta}+\tau^{2} \bar{\delta}^{2}+\bar{\delta}^{2} \tau \geq 1-2 \tau \bar{\delta}+\tau^{2} \bar{\delta}^{2}\left(1+\frac{1}{\tau}\right) \geq 1-2 \tau \bar{\delta}+2 \tau^{2} \bar{\delta}^{2} \geq \frac{1}{2},
$$

where the last inequality comes from the property $\tau \bar{\delta} \in(0,1]$. Thus we get

$$
h(\alpha, \beta, \bar{\delta}) \geq \frac{1}{\sqrt{2}} .
$$

Subcase 3.b. The last case is for $\bar{\delta}>\tau^{-1}$. Then we can rewrite

$$
\begin{aligned}
h(\alpha, \beta, \bar{\delta}) & =-\sqrt{(1-\tau \bar{\delta})^{2}}+\sqrt{(1-\tau \bar{\delta})^{2}+\bar{\delta}^{2} \beta^{2}(1+2 \alpha)^{2}} \\
& \geq-\sqrt{(1-\tau \bar{\delta})^{2}}+\sqrt{(1-\tau \bar{\delta})^{2}+\bar{\delta}^{2} \beta^{2}} .
\end{aligned}
$$

From (B.5) and the subcase assumption, we get successively

$$
\bar{\delta} \beta^{2}>\frac{\beta^{2}}{\tau} \geq 1
$$

Thus,

$$
\begin{aligned}
h(\alpha, \beta, \bar{\delta}) & \geq-\sqrt{(1-\tau \bar{\delta})^{2}}+\sqrt{(1-\tau \bar{\delta})^{2}+\bar{\delta}} \\
& \geq \frac{\bar{\delta}}{\sqrt{(1-\tau \bar{\delta})^{2}}+\sqrt{(1-\tau \bar{\delta})^{2}+\bar{\delta}}} \geq \frac{\bar{\delta}}{2 \sqrt{(1-\tau \bar{\delta})^{2}+\bar{\delta}}} .
\end{aligned}
$$

On the other hand, from (B.5) and the subcase assumption and since $\bar{\delta}^{2} \geq \bar{\delta} \geq 1$, we have successively

$$
(1-\tau \bar{\delta})^{2}+\bar{\delta} \leq 1+\tau^{2} \bar{\delta}^{2}+\bar{\delta} \leq 1+\left(\tau^{2}+1\right) \bar{\delta}^{2} \leq 1+2 \bar{\delta}^{2} .
$$

Thus finally, and since $\bar{\delta}>1$, we have

$$
h(\alpha, \beta, \bar{\delta}) \geq \frac{\bar{\delta}}{2 \sqrt{1+2 \bar{\delta}^{2}}} \geq \frac{1}{2 \sqrt{3}} .
$$

Acknowledgements. Research of B. Boutin was partially supported by ANR project NABUCO, ANR-17-CE40-0025 and by the Innovative Training Networks (ITN) grant 642768 (ModCompShock). 


\section{REFERENCES}

[1] S. Benzoni-Gavage and D. Serre, Multi-Dimensional Hyperbolic Partial Differential Equations: First-Order Systems and Applications. Oxford University Press, Oxford (2007).

[2] C. Besse, P. Noble and D. Sanchez, Discrete transparent boundary conditions for the mixed KDV-BBM equation. J. Comput. Phys. 345 (2017) 484-509.

[3] G.-Q. Chen, C.D. Levermore and T.-P. Liu, Hyperbolic conservation laws with stiff relaxation terms and entropy. Commun. Pure Appl. Math. 47 (1994) 787-830.

[4] J.F. Clarke, Gas dynamics with relaxation effects. Rep. Prog. Phys. 41 (1978) 807-864.

[5] P. Colella, A. Majda and V. Roytburd, Theoretical and numerical structure for reacting shock waves. SIAM J. Sci. Stat. Comput. 7 (1986) 1059-1080.

[6] R.B. Guenther and J.W. Lee, Partial Differential Equations of Mathematical Physics and Integral Equations. Dover Publications, Inc., Mineola, NY (1996). Corrected reprint of the 1988 original.

[7] B. Gustafsson, H.-O. Kreiss and J. Oliger, Time Dependent Problems and Difference Methods, 2nd edition. John Wiley \& Sons, Hoboken, NJ (2013).

[8] B. Hanouzet and R. Natalini, Global existence of smooth solutions for partially dissipative hyperbolic systems with a convex entropy. Arch. Ration. Mech. Anal. 169 (2003) 89-117.

[9] S. Jin, Efficient asymptotic-preserving (AP) schemes for some multiscale kinetic equations. SIAM J. Sci. Comput. 21 (1999) $441-454$.

[10] S. Jin and Z. Xin, The relaxation schemes for systems of conservation laws in arbitrary space dimensions. Commun. Pure Appl. Math. 48 (1995) 235-276.

[11] H.-O. Kreiss and G. Scherer, Finite element and finite difference methods for hyperbolic partial differential equations. In: Mathematical Aspects of Finite Elements in Partial Differential Equations. Academic Press (1974) 195-212.

[12] H.-O. Kreiss and G. Scherer, On the existence of energy estimates for difference approximations for hyperbolic systems. Technical report, Uppsala University, Department of Scientific Computing, Uppsala, Sweden, 01 (1977).

[13] T.-P. Liu, Hyperbolic conservation laws with relaxation. Commun. Math. Phys. 108 (1987) 153-175.

[14] T. Myint-U and L. Debnath, Linear Partial Differential Equations for Scientists and Engineers, 4th edition. Birkhäuser, Basel (2007).

[15] J.J. Stoker, Water Waves. The Mathematical Theory with Applications. Reprint of the 1957 original. Wiley, New York, NY (1992).

[16] B. Strand, Summation by parts for finite difference approximations for $d / d x$. J. Comput. Phys. 110 (1994) $47-67$.

[17] J.C. Strikwerda, Finite Difference Schemes and Partial Differential Equations, 2nd editon. Society for Industrial and Applied Mathematics (SIAM), Philadelphia, PA (2004).

[18] L.N. Trefethen, Spectral Methods in Matlab. SIAM, Philadelphia, PA 10 (2000).

[19] L.N. Trefethen and J.A.C. Weideman, The exponentially convergent trapezoidal rule. SIAM Rev. 56 (2014) $385-458$.

[20] G.B. Whitham, Linear and Nonlinear Waves. John Wiley \& Sons, Hoboken, NJ (1974).

[21] Z. Xin and W.-Q. Xu, Stiff well-posedness and asymptotic convergence for a class of linear relaxation systems in a quarter plane. J. Differ. Equ. 167 (2000) 388-437.

[22] W.-A. Yong, Boundary conditions for hyperbolic systems with stiff source terms. Indiana Univ. Math. J. 48 (1999) $115-137$.

[23] W.-A. Yong, Singular perturbations of first-order hyperbolic systems with stiff source terms. J. Differ. Equ. 155 (1999) 89-132.

[24] W.-A. Yong, Entropy and global existence for hyperbolic balance laws. Arch. Ration. Mech. Anal. 172 (2004) $247-266$. 